Ambiances

anbiances Environnement sensible, architecture et espace urbain

$3 \mid 2017$

Animer l'espace public ? Entre programmation urbaine et activation citoyenne

\title{
La mémoire partagée de « la vie de café » dans les quartiers populaires centraux d'immigration
}

The memory of "cafés's life" in popular districts down town of immigration

\section{Catherine Gauthier}

\section{OpenEdition}

\section{Journals}

Édition électronique

URL : http://journals.openedition.org/ambiances/970

DOI : 10.4000/ambiances. 970

ISSN : 2266-839X

Éditeur :

Direction Générale des Patrimoines - DAPA - MCC, UMR 1563 - Ambiances Architectures Urbanités (AAU)

\section{Référence électronique}

Catherine Gauthier, «La mémoire partagée de « la vie de café » dans les quartiers populaires centraux d'immigration », Ambiances [En ligne], 3 | 2017, mis en ligne le 10 décembre 2017, consulté le 08 janvier 2020. URL : http://journals.openedition.org/ambiances/970 ; DOI : 10.4000/ambiances.970

Ce document a été généré automatiquement le 8 janvier 2020 .

Ambiances is licensed under a Creative Commons Attribution-NonCommercial-NoDerivatives 4.0 International License. 


\title{
La mémoire partagée de « la vie de café » dans les quartiers populaires centraux d'immigration ${ }^{1}$
}

The memory of "cafés's life" in popular districts down town of immigration

\author{
Catherine Gauthier
}

1 Saint-Étienne a connu une croissance urbaine à partir d'une agglomération de bourgs en proximité géographique, qui dès le XVIII siècle donnent à la ville des allures de regroupement de villages, autour d'un centre bourgeois. Au XIX ${ }^{e}$ siècle un développement économique et urbain exceptionnel attire des migrants de toute la France, des pays frontaliers d'Europe puis des colonies. Les industries sont fortement imbriquées au tissu urbain parsemé de puits de mine, de nombreuses fabriques et usines proches du centreville. Les quartiers de la ville intra-muros ont gardé les traces d'une structure éclatée en petites centralités aux spécialisations économiques et sociologiques encore visibles. La ville et le bassin houiller connaissent une crise progressive dès les premiers accords européens autour du commerce du charbon puis suite aux chocs pétroliers des années 1970. A leur fermeture, les mines et usines ont laissé des friches que la ville en déprise urbaine et démographique résorbe progressivement. Dans ce tissu urbain inégal et fragilisé, les cafés populaires sont perçus tantôt comme emblèmes de la mémoire collective populaire, tantôt comme établissements interlopes occultés dans les recoins d'une mémoire des « bas-fonds ».

2 Fortement liés à la vie ouvrière des années industrielles, ces estaminets sont aujourd'hui en voie de transformation, voire de disparition. Les bars semblent cependant avoir gardé la mémoire des activités qui ont marqué les quartiers dans lesquels ils sont situés. On peut se demander s'ils portent toutefois la mémoire de ces vagues migratoires et de leur accueil dans les quartiers populaires. En quoi et comment les cafés ont-ils été des lieux de solidarité pour l'immigration locale? Comment l'ambiance actuelle en témoigne? Comment faire le lien entre la mémoire des ambiances passées et les caractères de celles d'aujourd'hui ? Telles sont les questions auxquelles cet article tente de répondre. 
3 Pour étudier la mémoire de l'immigration dans les quartiers populaires des centres anciens de cette ville post-industrielle en reconversion, j'ai choisi entre juillet 2014 et septembre 2015 de poser mon enregistreur sur le zinc des cafés de ces quartiers centraux cosmopolites, accompagnée de la photographe Sandrine Binoux ${ }^{2}$. Son travail s'attachait à réaliser des portraits sensibles des gens et des lieux, à capter par l'image les ambiances à l'intérieur comme au seuil de chaque établissement. Entretiens et clichés ont ensemble produit le film photographique Sociologie de comptoirs, cafés cosmopolites ${ }^{3}$, qui traite des cafés de quartier comme lieux-mémoire de l'accueil de générations successives de migrants de diverses origines, arrivées à Saint-Étienne dans la deuxième moitié du XX ${ }^{\mathrm{e}}$ siècle (Gauthier, 2016).

4 L'enquête ethnographique auprès des patrons, serveurs et clientèles des cafés situés dans cinq quartiers du sud de la ville, rend compte des traces encore vivantes d'une culture de la mixité socioculturelle, plus ou moins bien comprise et assumée aujourd'hui.

Illustration 1 : Carte des quartiers de Saint-Étienne et cafés étudiés

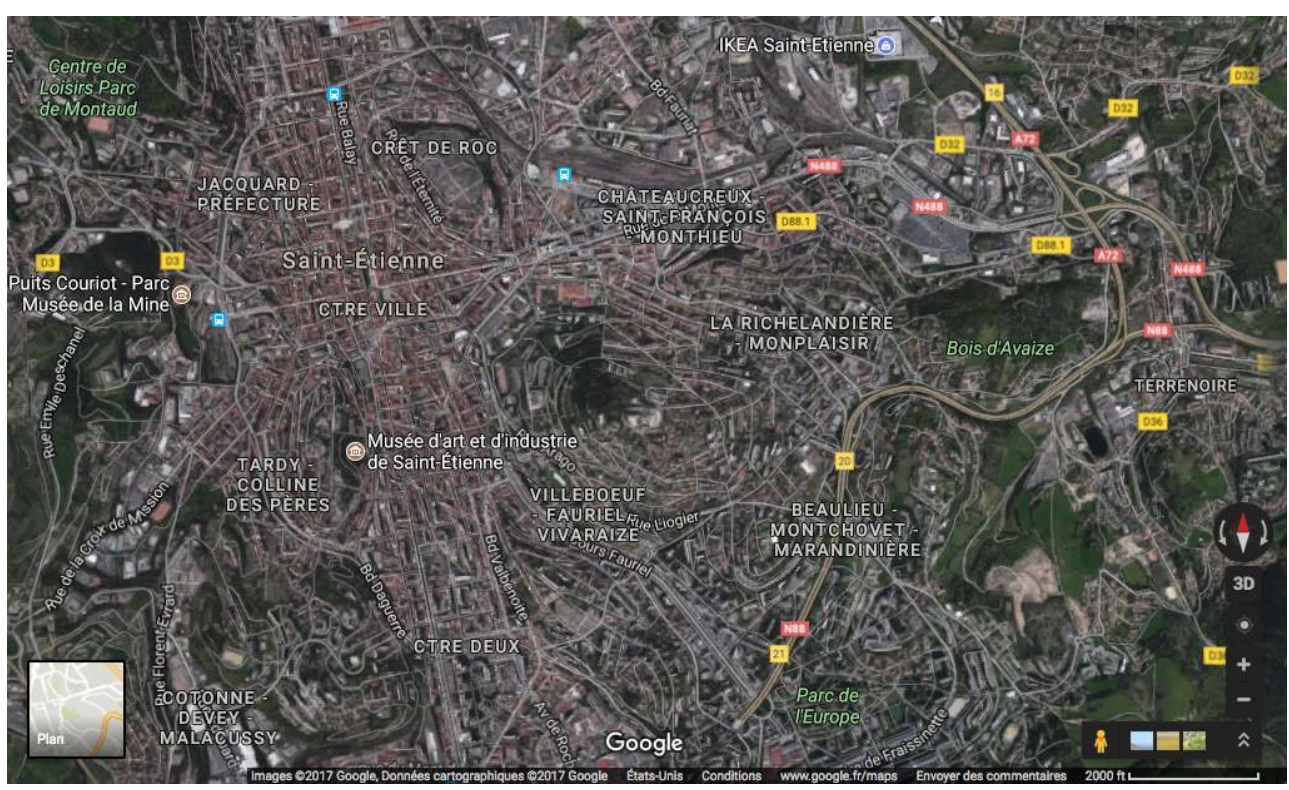

Source et copyright : Google Maps : https://www.google.com/maps/@45.42541,4.40909,4521m/ data $=! 3 \mathrm{~m} 1 ! 1 \mathrm{e} 3$

La démarche adopte ce principe énoncé par Michel Verret :

«Etudier une classe sociale par les lieux qu'elle occupe et n'occupe pas le temps qu'elle y passe et n'y passe pas, ce qu'elle y fait et n'y fait pas - qu'il s'agisse de l'usine ou des champs, du café ou de la maison, de l'école ou de la rue, de l'opéra ou de la prison. » (Verret, 1995)

6 Parmi ces "lieux qu'elle occupe ", les cafés sont appréhendés tant à partir de leur fonction commerciale et leur rôle social, que par leurs formes, leurs ambiances et leurs environnements.

7 Nous verrons à travers l'exemple de Saint-Étienne, comment les établissements cosmopolites des quartiers populaires contribuent à l'animation de villes postindustrielles qui tentent, à travers leurs aménageurs, de requalifier les formes urbaines par la «marchabilité » ou la « ludicité » des rues, places et friches (Cunier, 2014) ${ }^{4}$ . Nous verrons enfin en quoi, les pratiques et tactiques observables aujourd'hui 
témoignent de formes de maintien d'un droit à exister des quartiers anciens (Bacqué \& Levy, 2007).

Illustration 2 : Marché des paysans place du Peuple, 1953

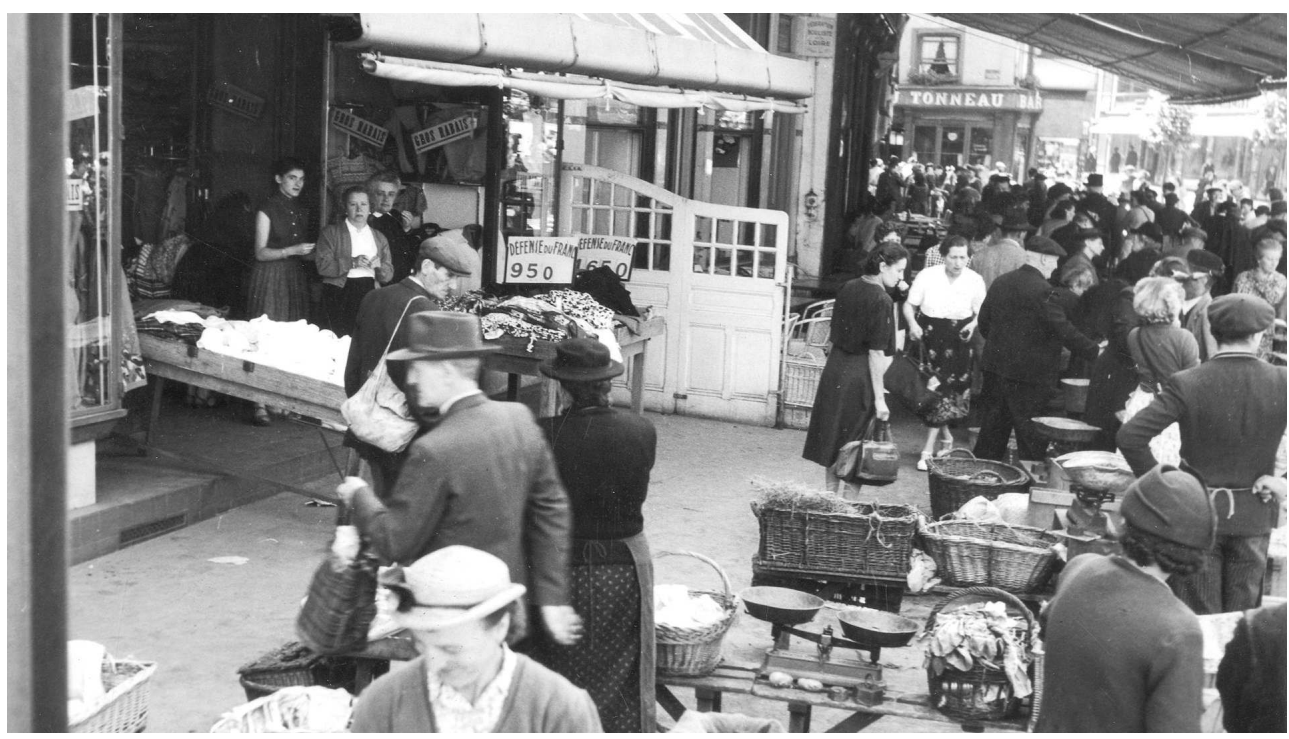

Source et copyright : Archives municipales de Saint-Étienne, 4 F 9 ICONO 2.

Illustration 3 : Des quartiers fortement chahutés, 1950

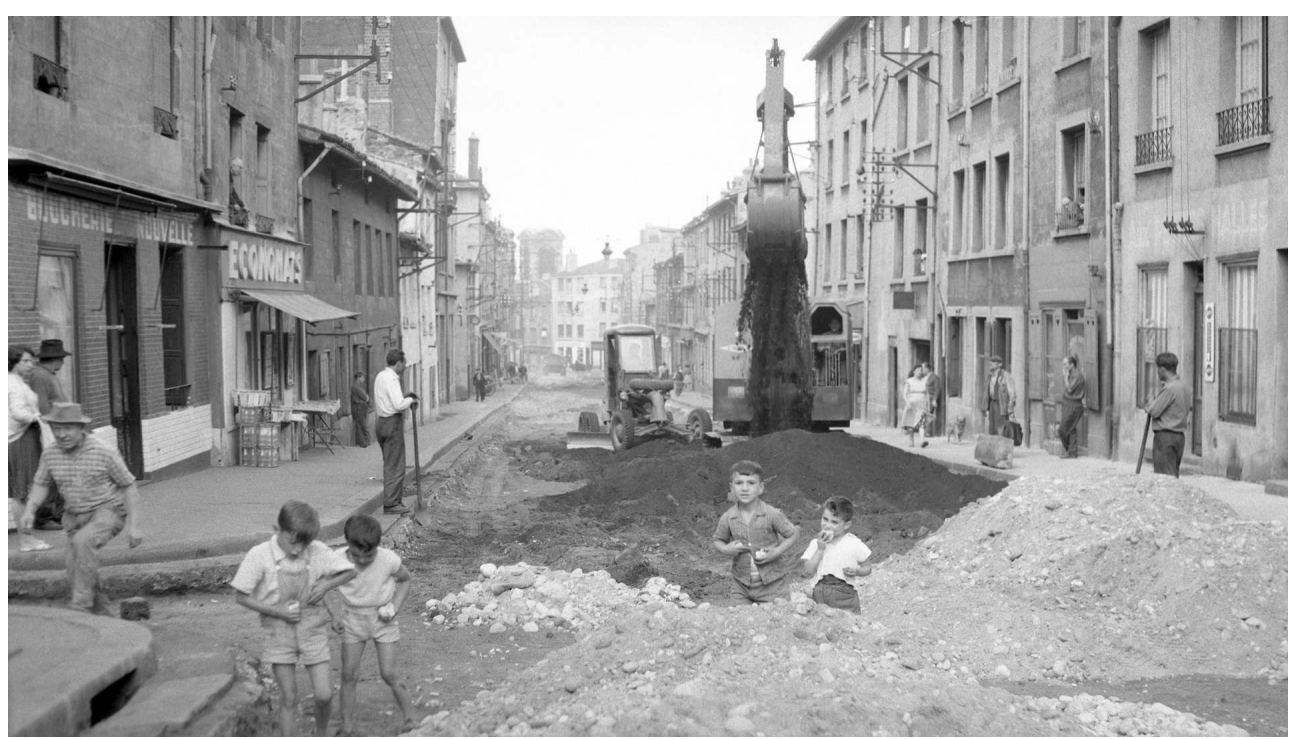

Source et copyright : (c) L. Leponce. Archives municipales de Saint-Étienne, 5 FI 7981

Parce que la diversité et les inégalités sociales, culturelles, religieuses, politiques peuvent se révéler dans l'étude des ambiances, il est important de regarder la ville comme Colette Pétonnet ${ }^{5}$ regarde le cimetière, en arpentant, furetant, sentant, en se laissant aborder par ses visiteurs, convaincre par ses usagers, s'en éloigner pour mieux y revenir... L'observation in situ a consisté ici à faire corps avec le terrain, éprouver ensemble avec ses occupants, l'espace, le lieu, la corporéité de l'ambiance (Thibaud, 2015; Marcellini \& Miliani, 1999), se placer dans les cadres de l'expérience qu'ils soient définis par les éléments naturels (pluie, soleil, froid, vent...) ou les rapports sociaux (retour d'un habitué, 
conflit avec un mauvais payeur, fête, repas en famille...). Et même si nos informateurs nous ont guidées, nous sommes aussi allées là où un café nous semblait particulièrement intéressant à intégrer à l'enquête: image extérieure, atmosphère, figure du patron, bouche-à-oreille.

9 En plus d'observations dans et aux abords de neuf cafés et une friterie, j'ai conduit des entretiens auprès de clients, patrons, serveurs. Des témoins «historiques » ont été interrogés sur le rôle des cafés auprès des immigrés en provenance de Kabylie, du Maroc, d'Espagne, du Portugal, de Turquie et d'Italie. Ils ont été sélectionnés selon la méthode du proche-en-proche pour favoriser une relation de confiance. Les recueils de témoignages en condition, d'entretiens biographiques selon un panel contrasté ${ }^{6}$ ont donné accès à des situations diversifiées et permis de multiplier les points de vue.

\section{La mémoire et l'expression contemporaine d'une culture vernaculaire entre espace ouvrier et migratoire}

10 La notion de quartier populaire est apparue après-guerre, pour valoriser un espace urbain central ancien, sa tradition d'accueil, d'entre-aide et de solidarité de classe, de convivialité simple, spontanée et démonstrative (Clerval, 2011). La transposition de pratiques rurales à l'espace urbain, du fait de l'afflux de populations extérieures et/ou étrangère, produit des usages populaires de la rue. Animés par les activités artisanales, industrielles et commerciales, les bars et cafés-restaurants sont les lieux emblématiques de la rencontre de l'altérité, ou de la promotion de la diversité sociale et culturelle.

\section{La notion de populaire dans un contexte stéphanois hétérogène}

On trouve dans les archives les clichés des photographes stéphanois Leponce ou Paul qui ont restitué au début et au milieu du $\mathrm{XX}^{\mathrm{e}}$ siècle ces ambiances quotidiennes auxquelles le film fait référence.

Illustration 4 : Marché aux Ursules 1950-1960

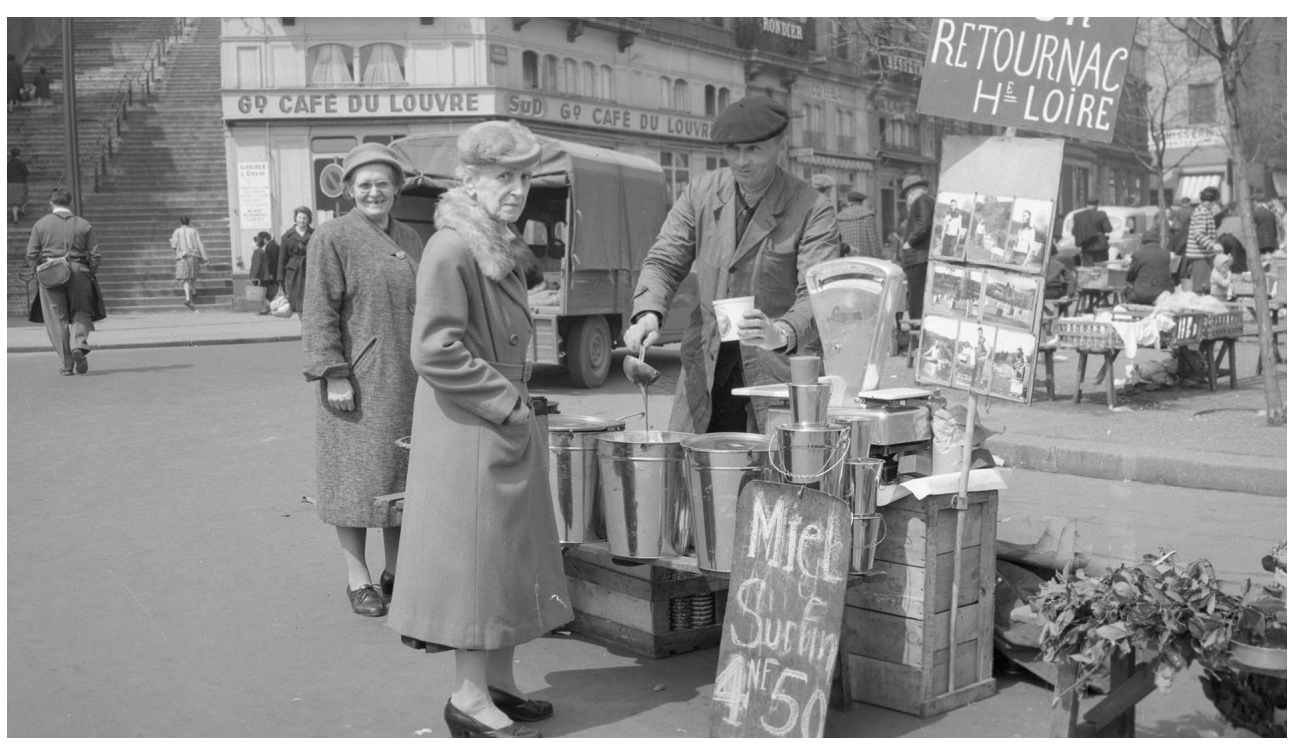

Source et copyright : (c) L. Leponce. Archives municipales de Saint-Étienne, 5 Fl 9269. 
12 devantures, passants et jeux d'enfants fabriquent l'imagerie des quartiers populaires situés dans les centres anciens. D'abord implantés aux marges des villes, ils assurent progressivement intra-muros, sous l'effet de la croissance urbaine, le statut de sas dans la ville. Ils accueillent aussi les migrants qui prennent part au peuplement de la ville et ainsi grossissent la proportion de la classe populaire dans la population (Gauthier, 2013; Gauthier, 2014 ; Siblot et al., 2015). Ce processus aboutit à des centralités résidentielles et commerciales pour les immigrés anciens ou nouvellement arrivés (Toubon \& Messama, 1990). Selon l'INSEE, en 1968, les ouvriers représentaient $43 \%$ de la population stéphanoise. Ils ne représentent plus qu'un quart des actifs en 2017, au profit des employés qui sont, eux, passés de $25 \%$ à $30 \%$.

13 A la croisée entre anthropologie de la mémoire migratoire ${ }^{7}$, socio-histoire du monde ouvrier et ethnologie urbaine des villes post-industrielles en mutation, la notion de « quartier populaire » (Sauvadet \& Bacqué, 2011) fait encore sens pour les observateurs et acteurs des transformations urbaines souvent pour esthétiser les termes de «pauvre » ou de « cosmopolite ».

dont nous parlons ici relèvent d'un cosmopolitisme ancien (Tarrius \& Misaoui, 2001), où la mixité et la rencontre sont depuis longtemps inscrites dans «l'identité du quartier ». Par-delà les désignations réductrices de "quartier arabe » ou " ghetto », la centralité commerciale immigrée manifeste « une appropriation ethnique particulière : sa vocation commerciale en fait un lieu d'attraction pour les citadins de même origine que les commerçants, et à divers titres (exotisme, affinités) pour les citadins de toutes origines» (Raulin, 2001 p. 177).

\section{Le café comme interface}

15 L'étroite relation entre le lieu de travail et le café est une des premières constantes repérées et exprimées par les enquêtés. Les cafés des quartiers populaires étaient d'abord ceux que les ouvriers, et les mineurs en particulier, fréquentaient avant ou après la prise de poste à l'atelier ou au fond. Mineurs et ouvriers se retrouvaient le matin pour prendre un petit marc, un rhum, avant que les véhicules des Houillères de la Loire s'arrêtent pour les conduire jusqu'au puits : «C'était sûrement pour se donner du courage! » interprète Morade $^{8}$, cafetier, fils d'un cafetier-mineur venu de Kabylie. 


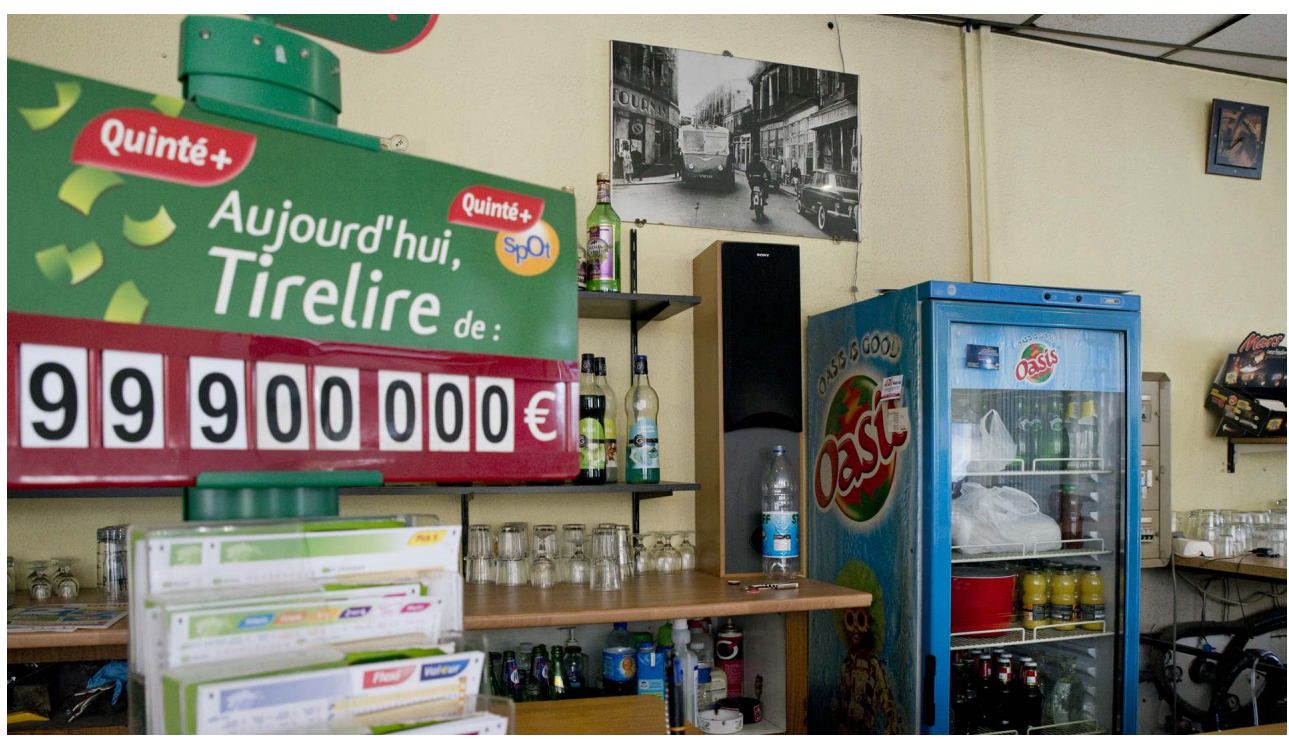

Source et copyright : ( ) Sandrine Binoux 2015.

«L'espace de résidence des ouvriers c'est non seulement l'espace d'enveloppement et de protection occupé et aménagé pour l'habitation du ménage ouvrier, mais aussi l'espace d'environnement proche et lointain, qui constitue à son tour l'enveloppe $\mathrm{du}$ premier et son horizon, et même ces espaces d'au-delà de l'horizon, dont l'exigence hante le souci quotidien des habitants (travail, trajets, démarches) ou dont l'espérance dessine les lignes de fuite de leurs rêves (dimanche, vacances, voyages)... » (Verret, 1995)

Dans les années 1970, voire 1980, les cafés-restaurants prennent encore une large part de la construction de la cohésion sociale locale. Sandra, la patronne du Bar des Acacias autrefois tenu par son oncle et avant par sa grand-mère mariée à un Marocain, sait redire ces choses : «Avant le bar surtout pour notre classe sociale, enfin la classe ouvrière quoi, ça restait accessible, on va dire, et ça faisait partie du plaisir que pouvaient s'octroyer les gens hein !» Ce à quoi Boumé, un habitué du bar, ami d'enfance de la patronne, répond : «c'est une forme d'intelligence on va dire le café. C'est vrai, on rencontre d'autres gens donc ça nous donne d'autres positions, d'autres pensées...» 


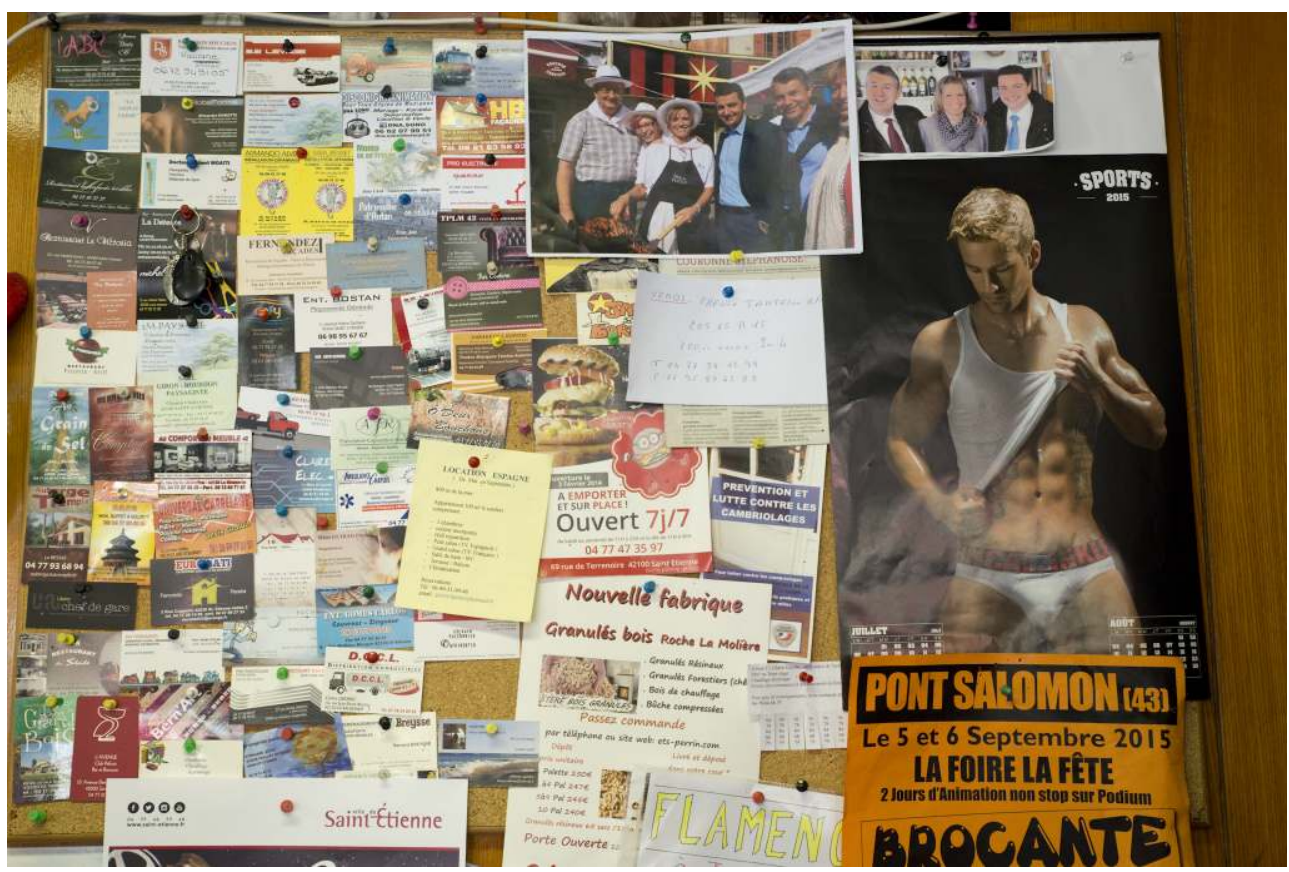

Source et copyright : ( ) Sandrine Binoux 2015.

Hier, le bar faisait l'interface ${ }^{9}$ entre la ville d'accueil et la terre d'origine. Brahim Highit, patron retraité d'un célèbre café kabyle aujourd'hui démoli, raconte à son tour :

« Moi mon bar s'appelait Sidi Aïch, le nom de mon village, c'est-à-dire les clients qui viennent de là-bas qui viennent d'Algérie, ils prennent l'adresse - Bar Sidi Aïch -, alors ils viennent et ils rentrent chez moi. "

Pour l'immigré, le café prend une dimension d'entre-deux. Mohamed Mamri, arrivé en France dans les années 1960, aujourd'hui militant associatif de la communauté kabyle locale, parle de son père et du rôle qu'ont tenu les cafés de Beaubrun, quartier central historique d'immigration :

« Arrivé là, il n'avait ni travail ni domicile fixe. Depuis notre arrivée quand même le lien que j'avais moi petit bambin de 10 ans avec les bistrots c'est que mon père m'amenait avec lui. Et on retrouvait pratiquement toutes les composantes du village. On continuait à parler sa langue donc, le kabyle, on continuait à parler des histoires de là-bas, c'est-à-dire des histoires de terrains, des histoires de ... l'argent tiens: qui c'est qui va partir là-bas pour que j'envoie 10 francs au bled? Etc. On restait toujours dans le contexte de là-bas. » 
Illustration 7 : Rue Tarentaize, années 1950

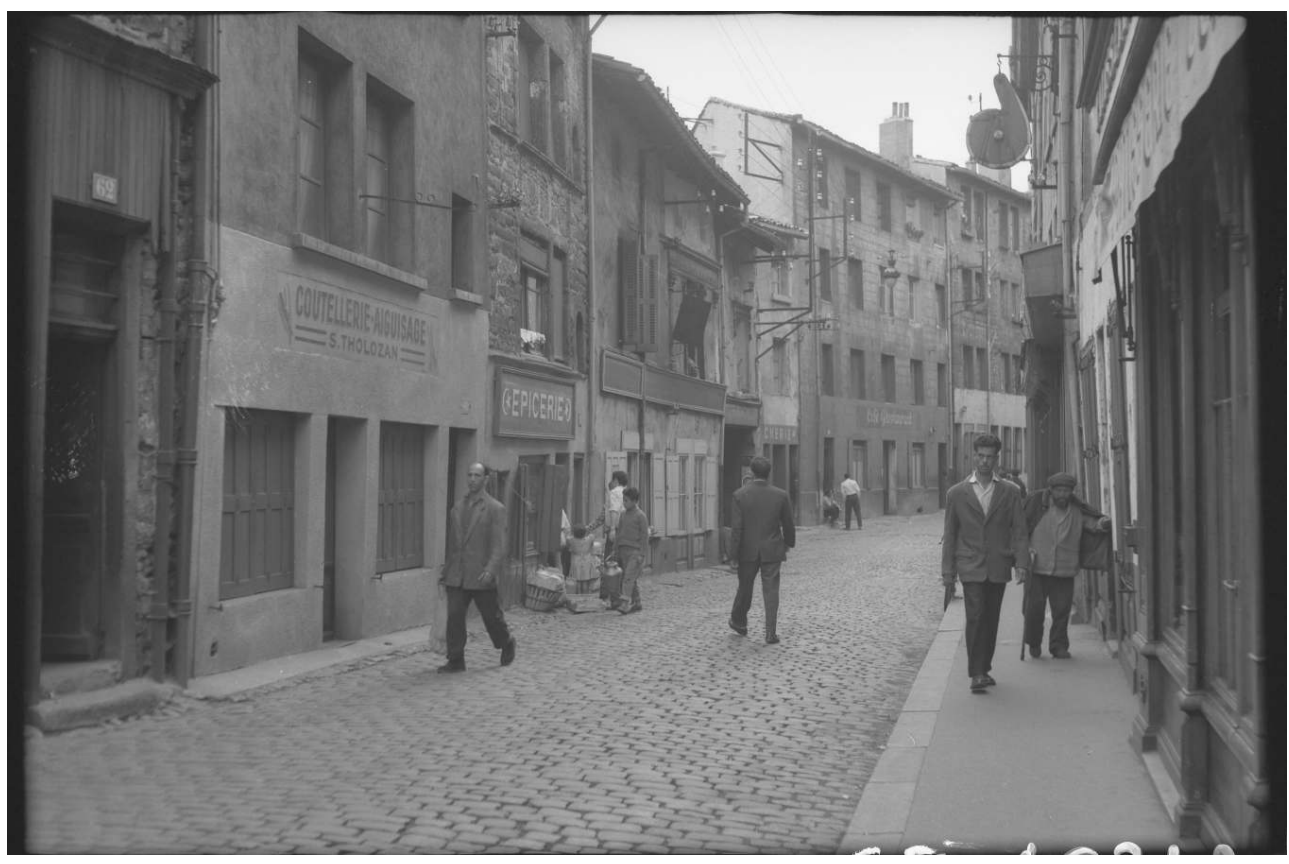

Source et copyright : (c) L. Leponce. Archives municipales de Saint-Étienne, 5FI 10347.

Illustration 8 : Le Bejaïa place de la Pareille aujourd'hui

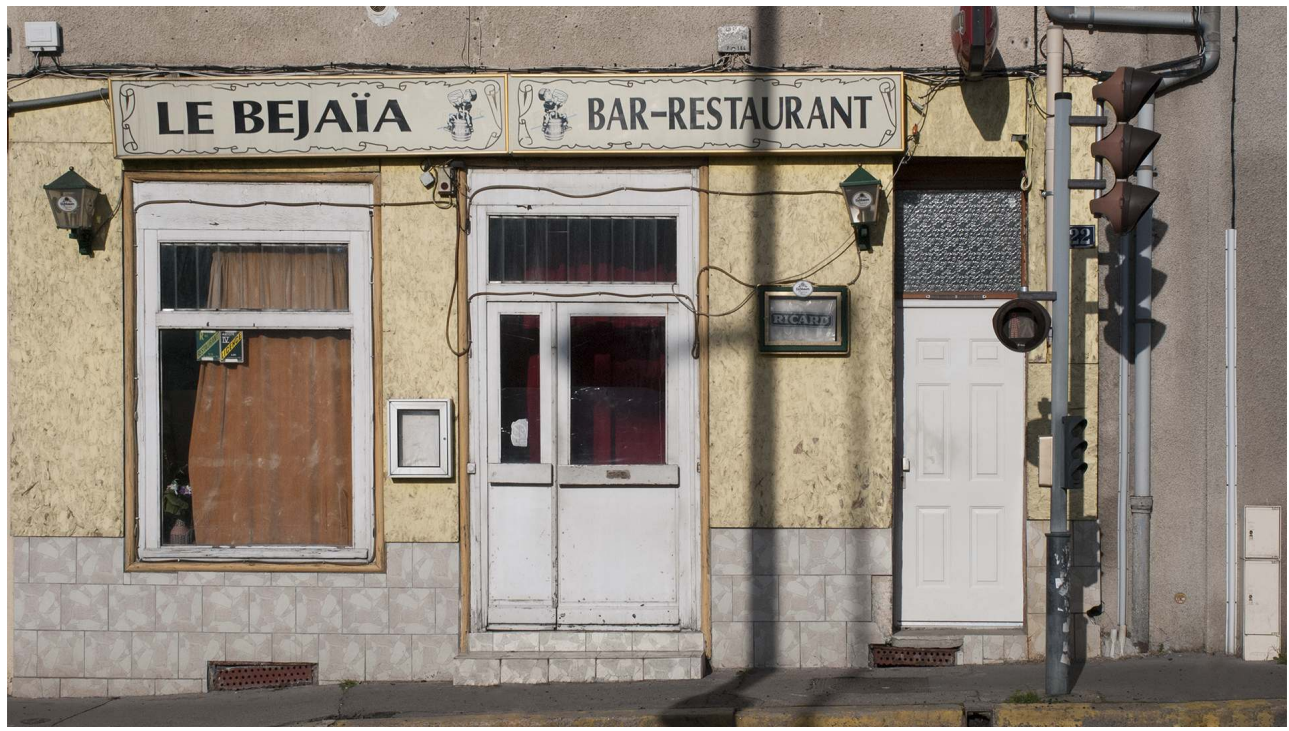

Source et copyright : ( ) Sandrine Binoux 2015

19 Aujourd'hui, la mixité exprime, au-delà du côtoiement, l'hybridation sociale et ethnique de ces quartiers. Morade, patron d'un PMU, prend très au sérieux son rôle de passeur entre société d'accueil et migrants : " à nous à faire que cette multitude de gens qui se connaissent pas, se parlent entre eux ». Comme Mourade, les autres interviewés placent la notion d'entraide au-dessus des conflits et cherchent souvent à exprimer le respect qu'ils ont pour leur clientèle traditionnelle. Marijo d'origine portugaise, qui tient un café à Terrenoire, ancien faubourg intégré aujourd'hui à la ville ${ }^{10}$, précise sa position face à ses deux clientèles : 
«Ça m'arrive de parler portugais mais j'évite parce que j'aime pas trop. C'est pas que j'aime pas le parler. C'est que quand il y a autour de nous des personnes qui le parlent pas, elles savent pas de quoi on parle, donc ça plaît pas forcément, alors j'évite..."

Ils offraient « un accueil où les gens venaient échanger un domino ou un jeu de cartes qu'on appelait la Ronda et ils faisaient office aussi de poste, de banquier, de recruteurs : je dirais que c'était pratiquement le travail temporaire de l'époque hein!» rappelle Mohamed Mamri. Aujourd'hui, lorsqu'ils sont investis de façon régulière et durable, ils garantissent un « capital d'autochtonie » (Rétière, 2003) non négligeable, a fortiori quand les origines locales sont à légitimer. Les « établis » (Siblot et al., 2015, p. 152-153 ; Tapie, 2014, p. 218-219), ceux qui ont grandi dans ce quartier, affirment cette appartenance. Il est important de se distinguer des plus précaires, nouveaux arrivés dans le quartier, sous peine de déclassement. Par cet ancrage local que donne l'appartenance à la clientèle d'un café et son implication dans les sociabilités du quartier, ils accèdent à des ressources symboliques et un entre soi nécessaire. D'où l'importance de l'évocation des souvenirs d'enfance qui témoignent de cette appartenance à une communauté d'expérience.

Illustration 9 : Roland montrant des photos des années 1920

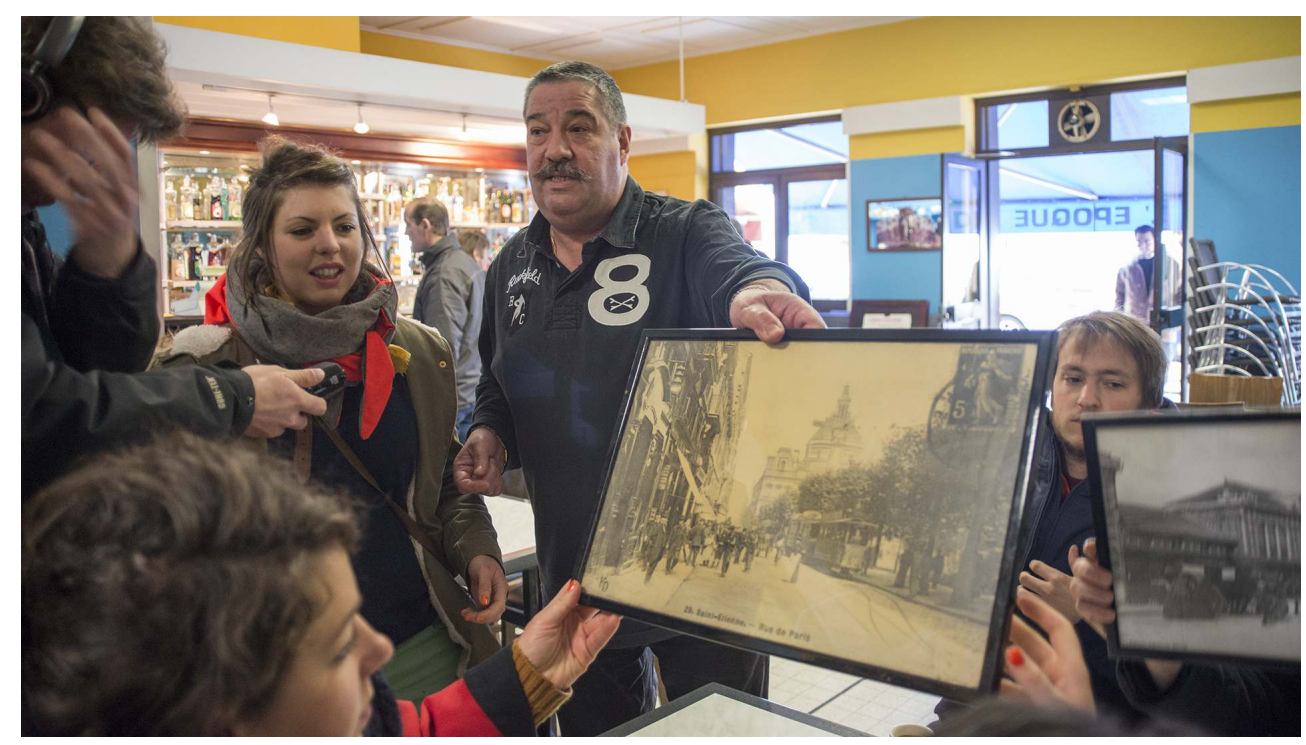

Source et copyright : (c) Sandrine Binoux 2015. 
Ce média ne peut être affiché ici. Veuillez vous reporter à l'édition en ligne http:// journals.openedition.org/ambiances/970

Source et copyright : ( c Le muséophone 2015. Avec l'aimable autorisation de Pierre-Vincent Fortunier.

\section{Le lieu d'une mémoire en acte}

24 Avec l'endormissement des chevalements des puits, les cheminées devenues muettes et le silence des petits ateliers, l'ambiance de la ville s'est profondément transformée. Les cafés demeurent néanmoins les lieux importants où cette histoire ordinaire quotidienne ${ }^{11}$ se poursuit, continue de se "révéler ", pour utiliser la métaphore photographique, et ainsi de se transmettre. Ils deviennent «lieu mémoire» au sens d'un lieu possible de patrimonialisation, d'un patrimoine qui cesse ici d'être silencieux (Hatzfeld, 2015). Ce ne sont pas véritablement des "lieux de mémoire ", au sens d'un Pierre Nora, conscients d'accomplir un "devoir de mémoire ", encore moins des lieux d'hommages officiels et stratégiques, mais plutôt des lieux d'exercice de la mémoire. Nous pourrions plus justement parler de " milieu de mémoire ", suivant en cela la proposition des sociologues qui ont enquêté sur les hôtels meublés parisiens :

"C'est comme milieu, autrement dit comme espace d'un groupe ayant en commun,

de façon plus ou moins marquée, une histoire, des références, des valeurs et un

mode de vie que nous avons saisi l'hôtel. » (Barrère \& Lévy-Vroelant, 2012, p. 10)

Les photos captent quant à elles, sur les murs des cafés, des reproductions de cartes postales anciennes de la ville côtoyant les photos des villages d'origine, exposées de façon ostentatoire en une affirmation identitaire duale. Fait d'autant plus marquant dans ces quartiers bouleversés par les restructurations urbaines successives. Dans ce décor souvent stigmatisé, les commerçants déploient des tactiques de survie, des stratégies commerciales soignant leur devanture, des agencements intérieurs, révélateurs des modes de transaction à la fois sociale et commerciale (Raulin,1986). Le cafetier qui désire transmettre un message d'acculturation réussie peut jouer ostensiblement des appartenances anciennes et nouvelles par exemple en accrochant un drapeau tricolore. 


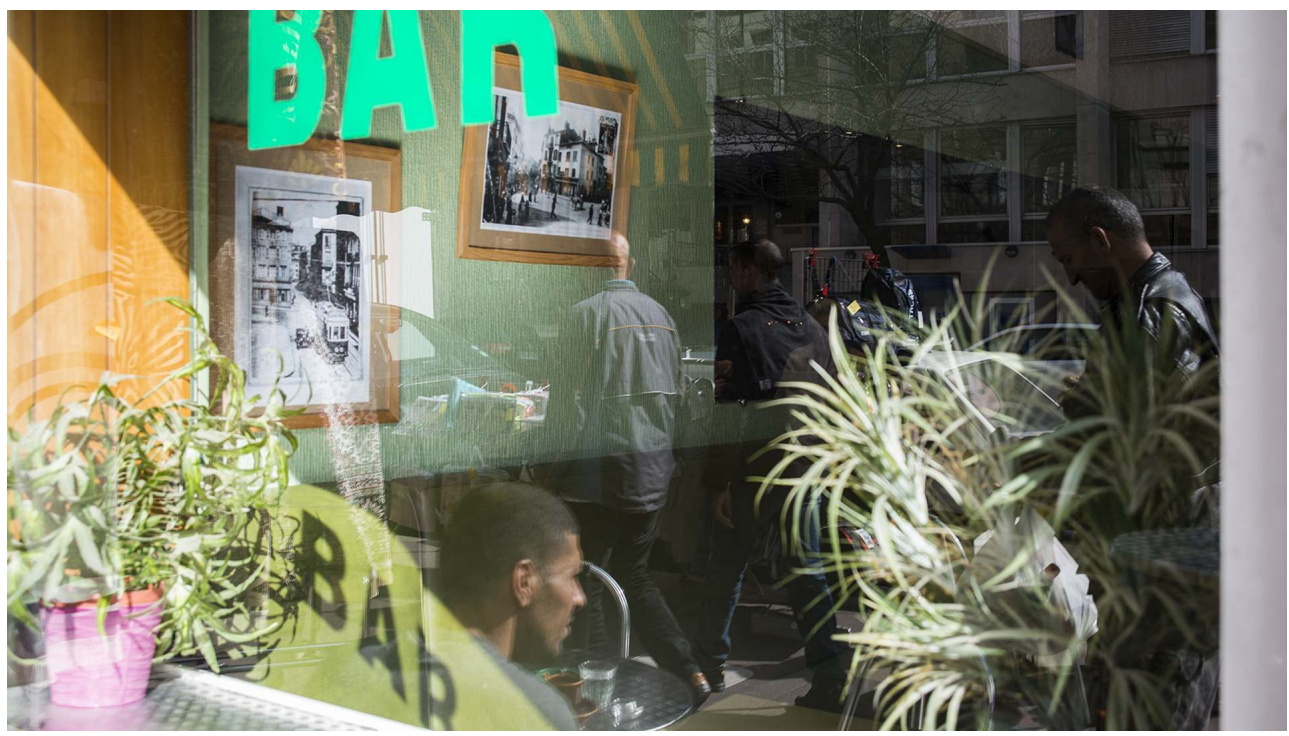

Source et copyright : ( ) Sandrine Binoux 2015.

Si le café continue d'appartenir à la sphère de l'habiter ouvrier, il est pris dans plusieurs espaces et échelles. «Habiter déborde les murs de la maison, facilite l'inscription active dans le monde à partir des mémoires, des souvenirs, des souhaits, des projets, d'abord vécus et imaginés dans des espaces et des temps singuliers » (Clavel, 2002). Peu nobles et pourtant patrimoine à la fois matériel et immatériel, ils demeurent des éléments-signes du paysage culturel local.

\section{Temporalités et ambiances associées, d'hier à aujourd'hui}

27 A l'intérieur des établissements, le sentiment d'être chez-soi passe par l'engagement des corps : la familiarité avec les volumes, les lumières, l'agencement des objets, le contact des matériaux, la décoration. Avant même la rencontre avec autrui, client ou commerçant, c'est la rencontre avec le lieu qui compte, la rencontre sensible et kinesthésique.

\section{Ressentir et transmettre le familier}

Dans le quartier de Terrenoire, le Café du Centre bénéficie de la lumière du soleil, qui vient se réfléchir toute la journée dans ses miroirs. Cette luminosité est tamisée par un plafond bas et les murs lambrissés inchangés depuis les années 1970. Les clients y sont très attachés. Seul le bar, la tireuse et le percolateur sont récents. Les pots de fleurs sur les tables en faux marbre, les orchidées sur un rebord de fenêtre, la cuisine qu'il faut traverser pour atteindre les toilettes, tout est impeccablement propre et rangé. Derrière le zinc, des photos de famille présentent Marijo et ses petits-enfants. 


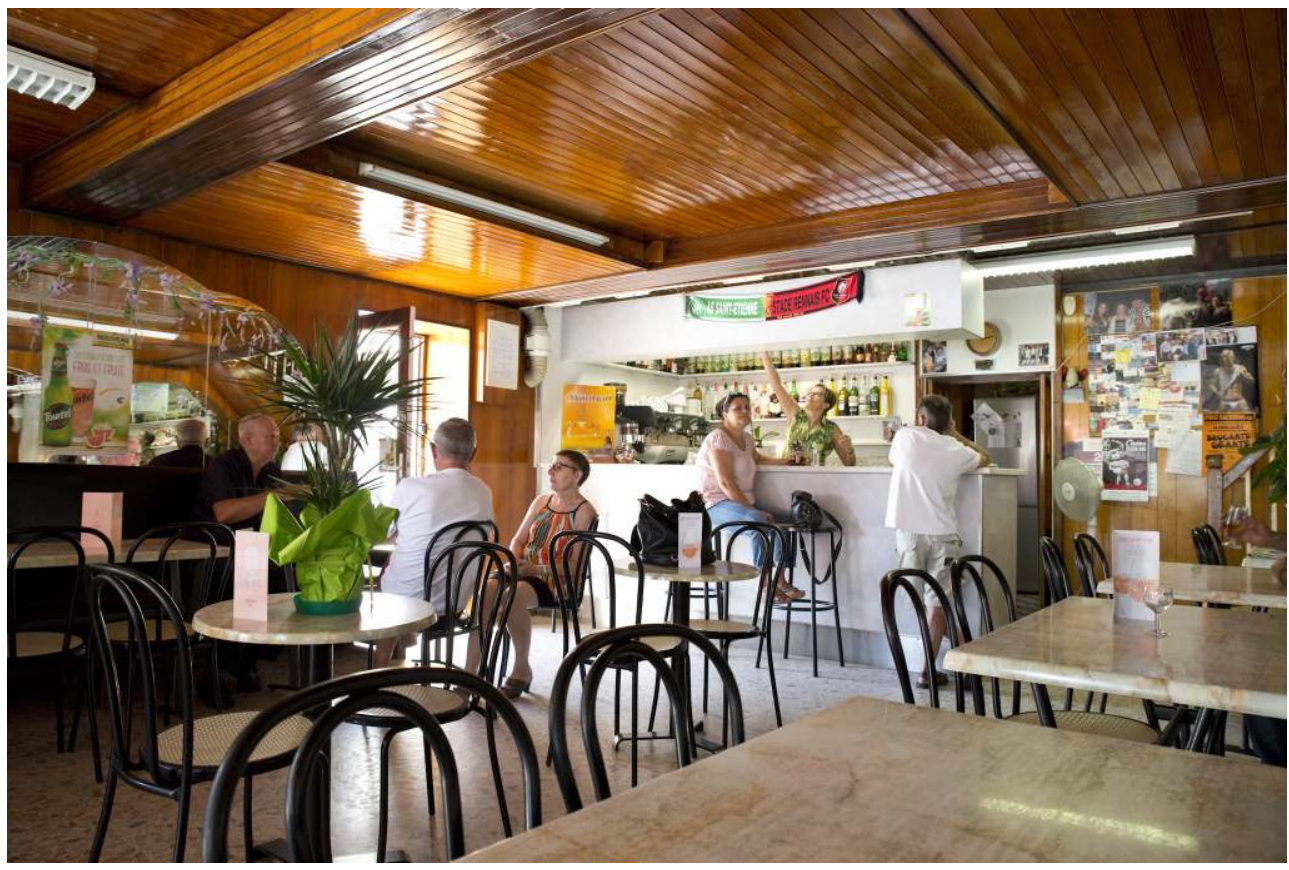

Source et copyright : ( ) Sandrine Binoux 2015.

Illustration 12 : Comme à la maison, la Friterie

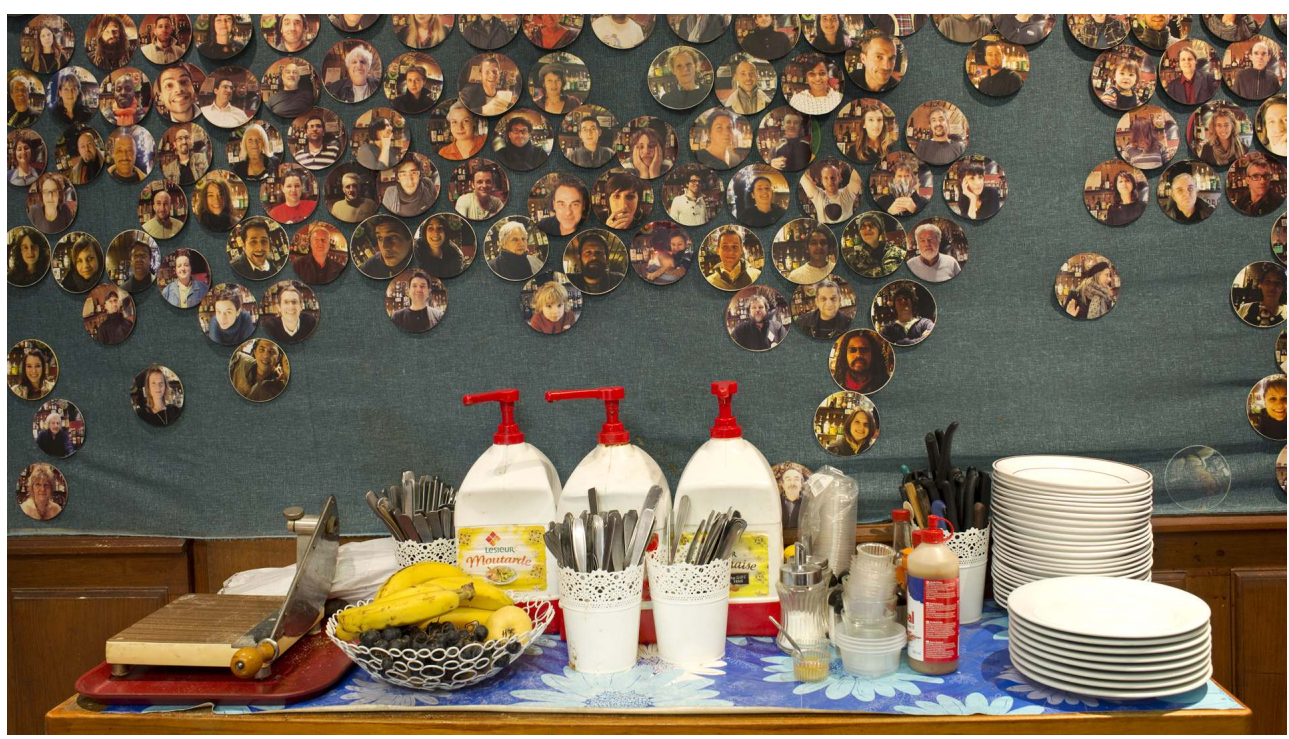

Source et copyright : ( ) Sandrine Binoux 2015.

29 A la Friterie, Mohamed et Louise ont toujours mis beaucoup de photos de famille sur la moquette bleu nuit des murs. Pour les quarante ans du lieu, leur fille a entrepris de photographier tous les clients volontaires et de coller ces photos sur des sous-verres accrochés ensuite sur le pourtour de la salle. Ces portraits rieurs et espiègles, aussi variés que la clientèle, forment ainsi une nébuleuse de visages qui côtoient sur le mur du fond les bouteilles de sauce mayonnaise et Ketchup posées sur la desserte des couverts. 
Illustration 13 : Souvenir des 40 ans de la Friterie avec les photos de famille

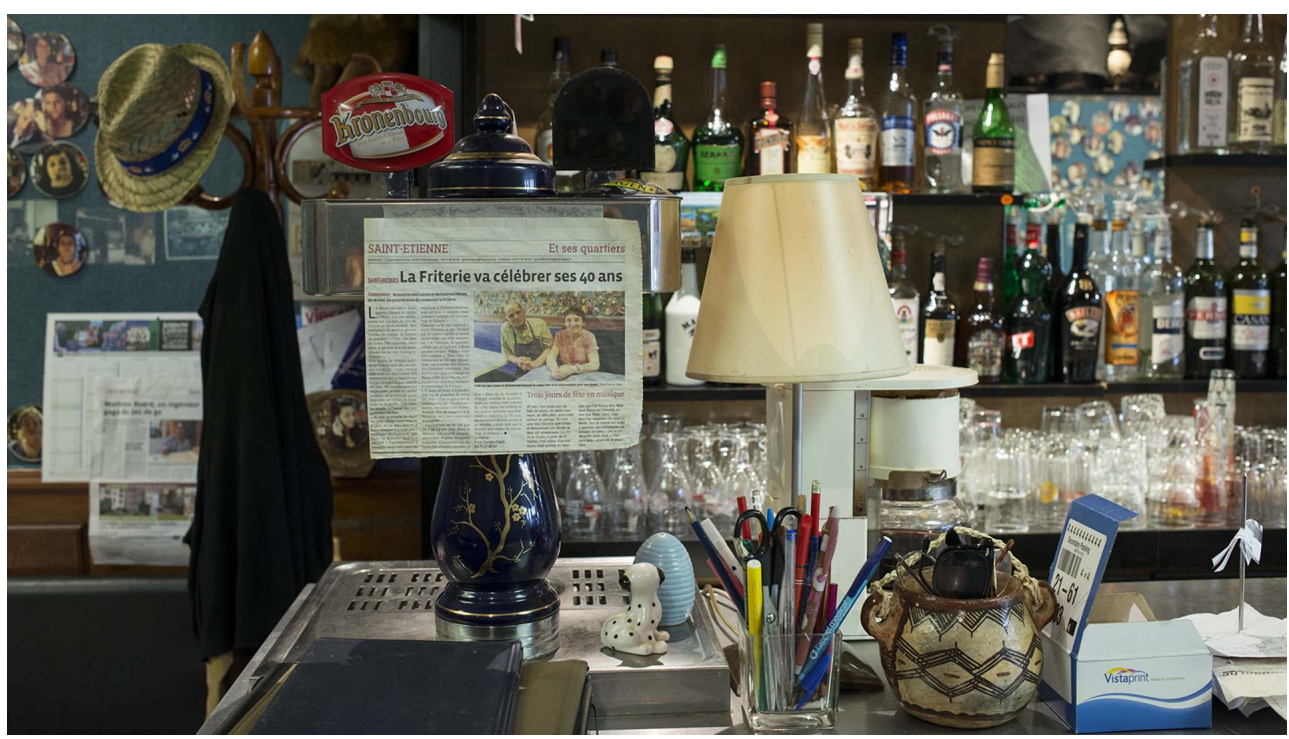

Source et copyright : ( ) Sandrine Binoux 2015.

Illustration 14 : Témoignages d'amitié au bar des Acacias

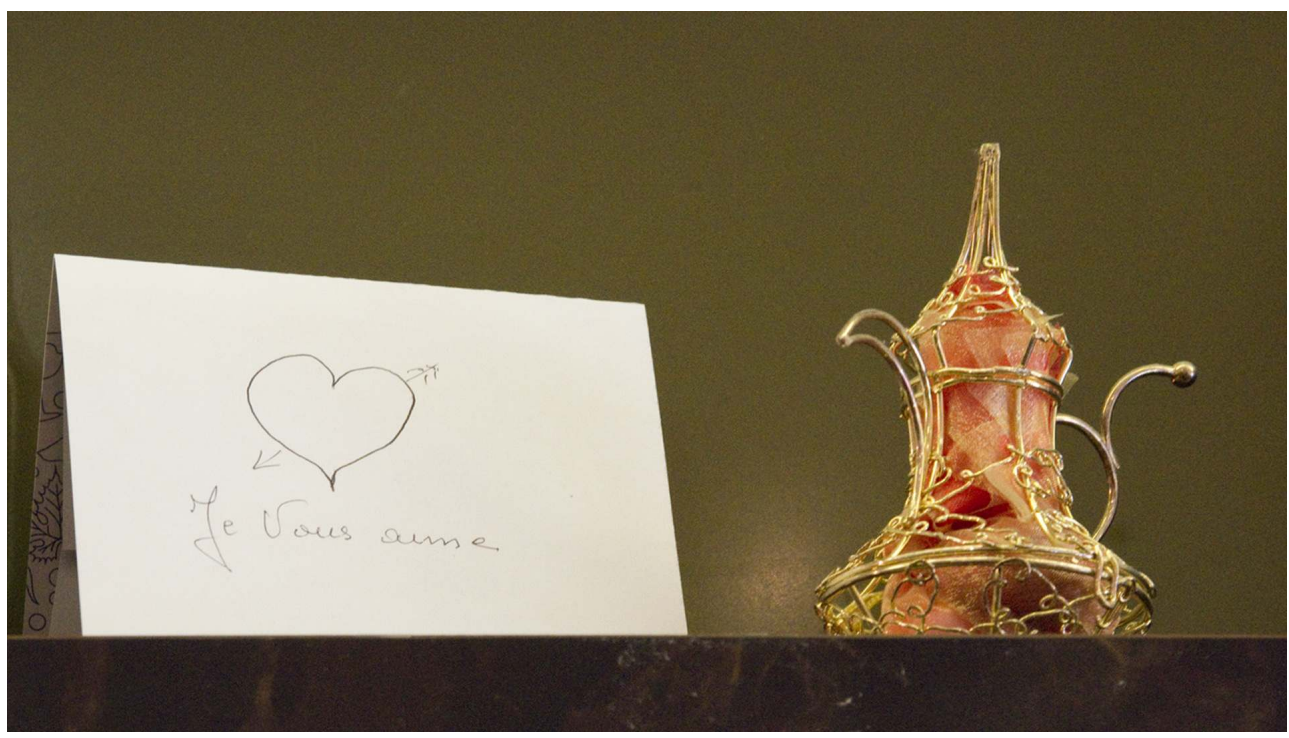

Source et copyright : (c) Sandrine Binoux 2015

Sur les rayonnages du bar des Acacias sont déposés des témoignages d'amitié des clients. Les banquettes vertes aux rondeurs accueillantes sont placées le long du mur face au zinc. $\mathrm{Au}$-dessus d'elles, de larges miroirs ouvrent l'espace. Près de la vitrine, une table baignée de lumière invite à la lecture solitaire tout en regardant l'animation de la rue et de la terrasse. Dans le fond, à la dernière table de la rangée proche de la petite salle privée, Sandra peut s'installer pour faire une partie de coinche avec les habitués ou éplucher ses légumes le jour du marché. 


\section{Les ambiances d'un lieu de ressources urbaines rythmé par la vie de quartier}

31 Tantôt abri contre les intempéries, tantôt endroit pour profiter du soleil, la luminosité du jour ou la profondeur de la nuit pénètrent son intérieur et l'âme de ses habitants. Les terrasses donnent une dimension saisonnière à la vie du café et le ton de la saison à la rue qui l'abrite. Lorsque le temps s'y prête, les clients s'installent pour observer le spectacle des passants (Pichon et al., 2015, planche 36).

32 L'ambiance des centralités urbaines immigrées n'est pas étrangère au fait que le centre peut naître de la marge et de la capacité des populations migrantes à faire quartier. Elles deviennent de véritables places du commerce distractif pour les plus démunis (Tarrius, 1997). L'exil et la pauvreté peuvent aussi faire convivialité. Ces ambiances ordinaires croisent les rythmes du quartier et de ses habitants, ceux du déplacement pendulaire vers le pays d'origine et ceux des heures, des semaines, voire des années égrainées au café (Thibaud, 2015, p. 24-29).

33 Les temporalités du café populaire sont celles de la vie ouvrière, des transports en commun, des courses de la ménagère, de la flânerie des passants. Avec la baisse de l'activité industrielle et des emplois correspondants, les rythmes actuels sont davantage ceux des retraités et des chômeurs : l'apéro quotidien, les jours de marché, le " petit noir » en début d'après-midi, les jeux de cartes et les concours de belote... La période du Ramadan offre quant à elle d'autres ambiances. Les horaires d'ouverture sont modifiés, les clients différents. Les cafés tenus par des Musulmans privilégient les horaires tardifs, après la rupture du jeûne par exemple, ou encore ferment durant toute la période. Chez ceux qui ont une clientèle importante de personnes pratiquantes sans l'être eux-mêmes comme Roland, le jour de l'Aïd n'est pas réellement fêté mais offre l'opportunité de " payer sa tournée». Occasion au cours de laquelle Roland aime glisser quelques mots d'arabe dans son vocabulaire fleuri pour saluer le retour de ses clients, disparus depuis quarante jours.

34 A toutes les époques, on vient écouter les artistes, eux-mêmes émigrés, souvent ouvriers, dans ces cafés devenant cabarets le soir. Les musiques de l'immigration se sont souvent créées en ces lieux, brodant sur l'exil, le travail et les conditions de vie (Suzanne, 2009). Boumé, qui très tôt accompagnait son père mineur au café, se souvient également:

« Je me revois dedans gamin à quatre ou cinq ans. Les cafés étaient remplis! Ça jouait de la guitare, mais c'est une image... Y'a ce monde un peu... L'odeur du vin, des cigarettes, le bavardage surtout. »

35 Au fil des années, s'accumule une expérience de «la vie de café » à laquelle certains, comme Boumé, ont été socialisés depuis leur jeune âge. Aujourd'hui, les patrons savent comment associer les clients à l'animation du lieu, quand calmer l'ambiance ou la stimuler, anticiper les commandes des habitués ou les ignorer à l'image du serveur décrit par Colette Pétonnet :

« Le contact professionnel possède à mes yeux une vertu apaisante. Ni artificiel ni servile mais réservé aux clients, il respecte l'anonymat. » (Pétonnet, 1987, p. 5)

Ce média ne peut être affiché ici. Veuillez vous reporter à l'édition en ligne http:// journals.openedition.org/ambiances/970

Source et copyright : ( \ Le muséophone 2015. Avec l'aimable autorisation de Pierre-Vincent Fortunier. 
A la croisée des dimensions physiques du lieu et des sociabilités, le climat ainsi généré est en partie conditionné par ce que patrons et habitués connaissent des propriétés sensibles de leur café et de son expérimentation continue, par exemple de ses qualités acoustiques ${ }^{12}$.

Illustration 15 : Départ en retraite d'un mineur années 1950-1960

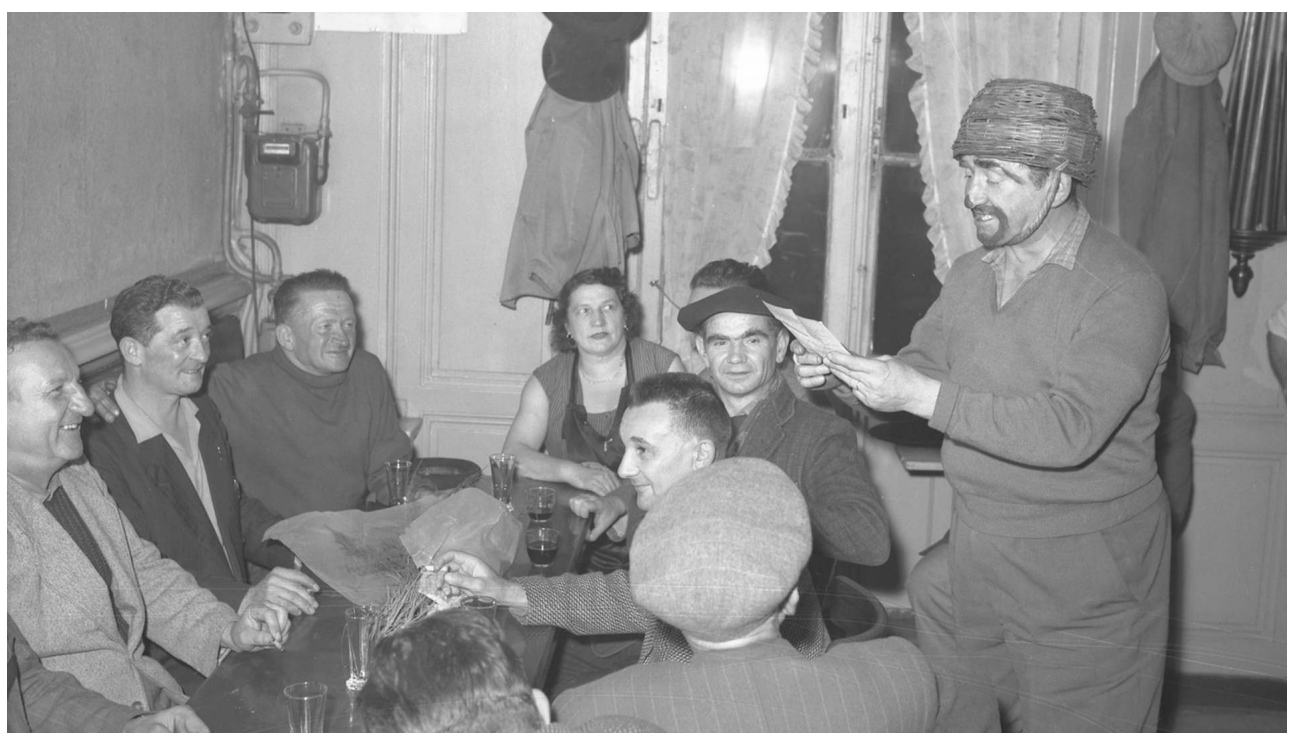

Source et copyright : (c) L. Leponce. Archives municipales de Saint-Étienne, 5FI 9072.

Dans le monde ouvrier et plus largement les classes populaires, les activités collectives sont explicitement valorisées dans les récits. Lorsque les patrons décrivent l'affluence des cafés entre les années 1950 et 1980, que les clients décrivent le chaud, le bruit, les coudeà-coude au bar, c'est aussi pour exprimer ce « demeurer en commun » de la vie sociale de la classe populaire, repliée aujourd'hui sur la vie domestique et familiale (Tapie, 2014, p. 218-219).

«C'est dommage que tu n'aies pas connu mon père (...). Lui il avait le mot et il avait le geste. Il pouvait passer derrière un papy et puis... lui faire un petit... tu vois (elle mime une accolade, une pression de la main sur le bras...). Il arrivait à percevoir le mec un peu triste ou désœuvré qui avait besoin non seulement d'entendre des paroles réconfortantes mais le fait de t'empoigner, du contact pour te dire : “bon ça va aller, on est là ". » (Sandra, troisième génération de cafetiers)

Ces espaces d'interconnaissance obéissent à une volonté du patron, des serveurs et de certains clients impliqués, de créer les conditions de l'échange, entre des personnes se rencontrant difficilement par ailleurs (Dacheux, 2003). Le café peut, en ce sens, être considéré comme un «espace social» au sens où l'entend Hannah Arendt: au-delà du domestique et en deçà de l'espace public, lieu où l'on se soumet au regard de l'autre et se laisse toucher par lui, lieu d'échange proche de l'intime (Arendt, 1983, 1958). 
Illustration 16 : Signe d'empathie

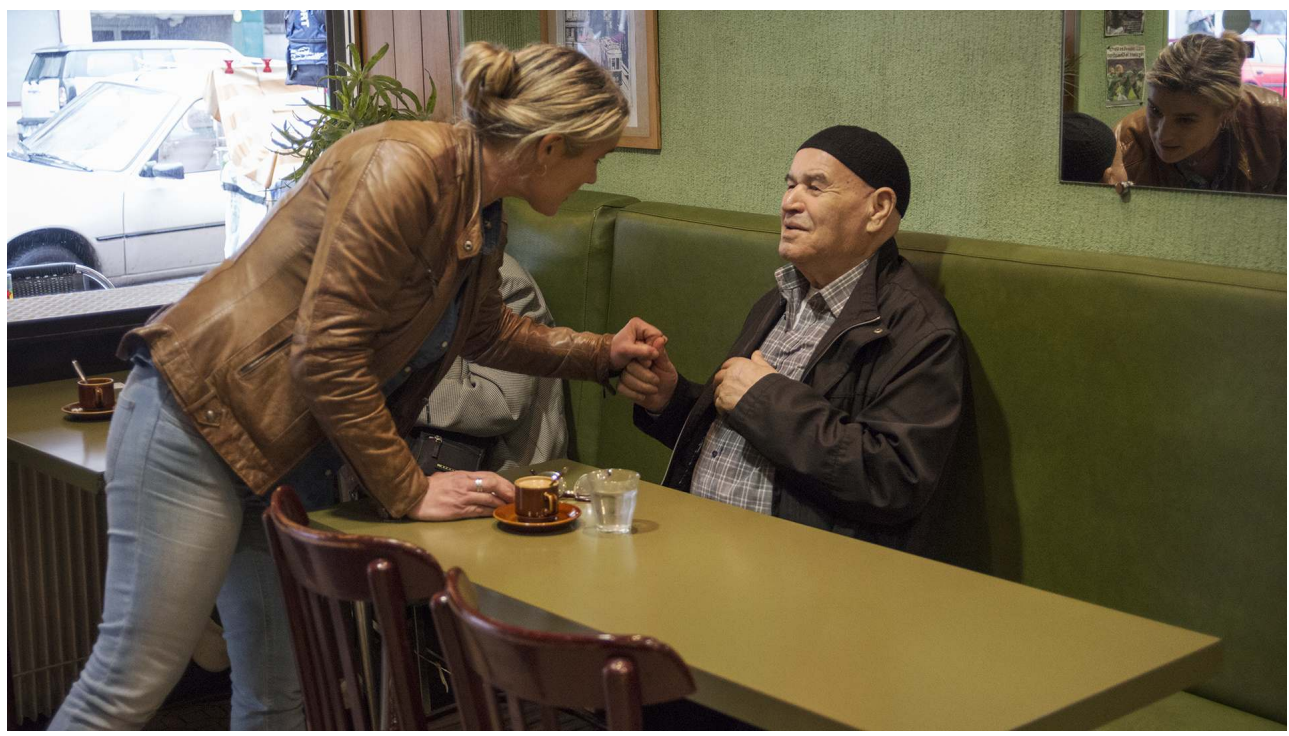

Source et copyright : ( ) Sandrine Binoux 2015.

Lieu d'échanges marchands, entre le commerce de proximité et le commerce distractif (Lemarchand, 2008), le café aménage une « ressource urbaine irremplaçable » au sein de la ville et ses aménités (Berroir et al., 2015). Des garnis ou bidonvilles des années 1930 aux grands ensembles de la périphérie des années 1960, les populations ont été déplacées. Malgré les démolitions et reconstructions, les fermetures d'usines, la disparition de certains métiers et activités commerciales et les tentatives de gentrification de ces quartiers, les cafés, les patrons et leur clientèle demeurent des références géographiques et sociales, des amarres relativement sûres pour des populations fragilisées par le chômage, la maladie de longue durée ou l'isolement dû à la vieillesse.

Illustration 17 : Client habitué du bar des Acacias

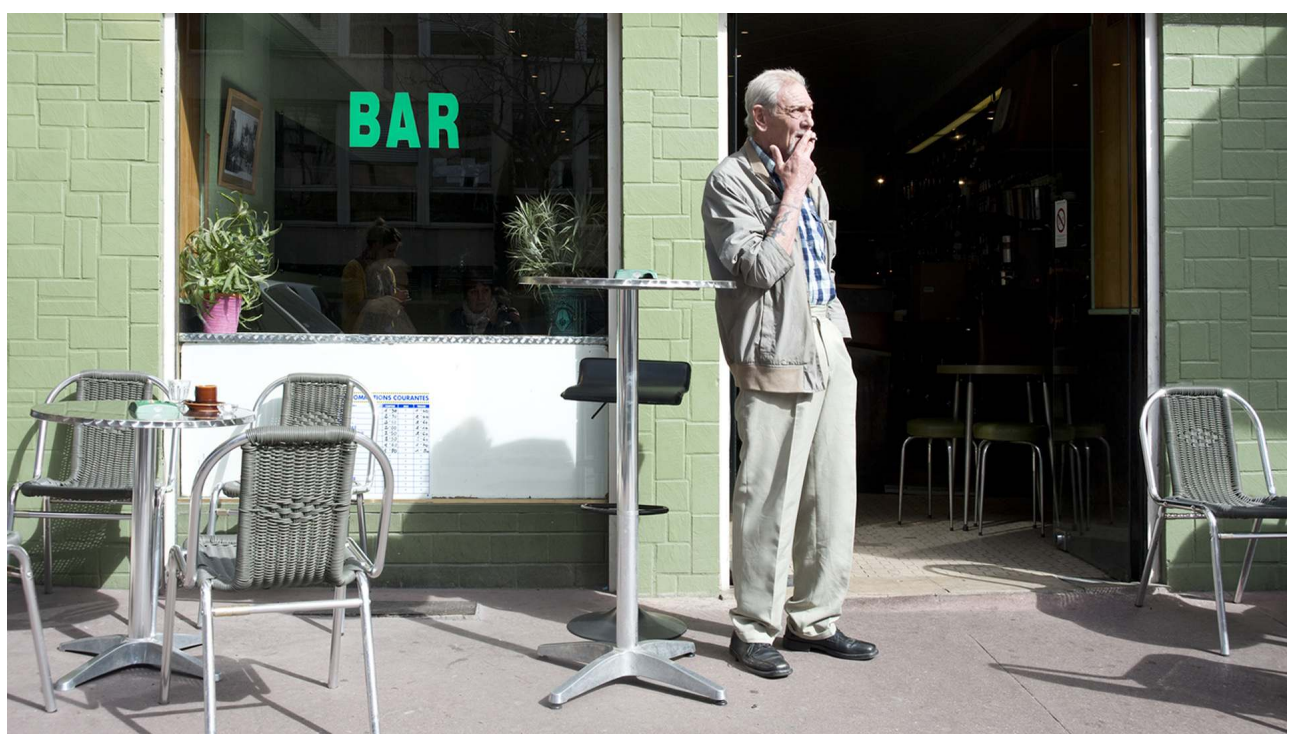

Source et copyright : ( C Sandrine Binoux 2015. 
Monsieur Benmamar, à plus de 90 ans, parcourt la moitié de la ville pour boire son café quotidien aux Acacias où ses amis sont sûrs de le rencontrer. Attendu comme un grandpère par la serveuse qui se laisse taquiner en prenant des nouvelles de sa santé et de celle de sa famille au pays, il fait avec humour la cour à Antonia, de deux ans son aînée.

Dans cette fidélité renouvelée, les populations se distribuent par affinité ethnique, générationnelle ou professionnelle, de voisinage ou d'amitié. Les enfants d'immigrés maghrébins connaissent le café car ils y accompagnaient souvent leur père enfant, faisant de cette sortie du quartier un moment d'intimité comme il y en avait peu.

«Je me souviens dans le quartier il y avait ceux qui allaient au bar et ceux qui n'y allaient pas. Parce que le bar il était quand même associé à un truc de jeu, de haram, d'illicite, de tout ça, je dirais. Mais aller dans un espace fermé, dans lequel les hommes jouent, etc., et là, où c'était des bars qui étaient souvent sur la place, plus ouverts, je pense que c'est pas tout à fait la même chose. » (Malika, assistante de service social, lieu d'entraide non loin de la Friterie)

Le climat, les qualités sensibles du lieu, sont des phénomènes localisés et situés dans le temps, que la recherche qualifie selon Jean-François Augoyard d' « ambiance in situ » qui s'analyse par la « reconnaissance des interdépendances entre forme construite, forme perçue et forme représentée ». Ainsi lorsque Malika distingue «cafés ouverts » et "cafés fermés", elle désigne «les propriétés physiques perceptibles, les instrumentations, fonctions, usages et façonnages affectant la forme construite et, enfin, l'ensemble des normes, règles, codes, références " (Augoyard, 2007, p. 37). Outre ses fonctions sociales quelques peu idéalisées, le bar demeure aujourd'hui un commerce de plaisir, de détente et de sociabilité.

Si comme le note Manuel Charpy à propos des échoppes du XVIII ${ }^{\mathrm{e}}$ siècle à Paris, «les marchands immergés dans la rue en connaissent tous les mouvements » (Charpy, 2011), les cafés implantés dans le quartier en connaissent toutes les respirations. Le café et sa clientèle exercent de tout temps une veille sociale sur les situations des clients les plus vulnérables dont l'absence prolongée alerte. Les patrons et leur personnel sont conscients de ce rôle. Ils se disent soucieux de rendre à leur clientèle des témoignages pour leur fidélité et leur confiance par l'organisation de distractions et d'événements festifs.

Les cafés sont donc des espaces intermédiaires (Bazin, 2009), entre le privé, la vie domestique et le public, la vie civile. On peut s'y sentir à la fois comme à la maison, parmi ses semblables et en même temps ce doit être un espace de tolérance et de confrontation à la différence, sociale, culturelle, politique, dans lesquels les patrons et serveurs exercent un rôle de médiateur parfois avec autorité.

\section{Espaces intermédiaires dans l'animation des places et des rues}

Petite société, échantillon de la ville comme espace de civilités, cet entre-soi de la communauté des clients est aussi celui de la pluralité et de l'altérité. Ce qui en fait un espace public au sens habermassien du terme: rassemblement populaire, pratique discursive et expression critique du politique. L'histoire des rapports entre les débits de boisson et la force publique est là pour rappeler à quel point ils suscitent depuis toujours la méfiance et font l'objet d'une surveillance étroite, pour les débats et réunions qu'ils peuvent abriter. Le mouvement ouvrier minier est en partie né dans les bistrots de la région stéphanoise et plusieurs anecdotes nous ont été rapportées sur des règlements de 
compte dans des cafés du quartier Beaubrun durant la guerre d'Algérie. L'espace public s'anime aussi par le conflit et le mouvement social passé.

\section{Changement social et urbain entre un âge d'or et un présent incertain}

Aujourd'hui, la notion de "quartier populaire " retrouve un regain d'intérêt dans le discours du projet urbain, pour vanter les qualités conviviales ou d'authenticité et la revalorisation esthétique de certains centres anciens (Giroud, 2011, p. 37-54). Une campagne de résorption des rez-de-chaussée vacants, encouragée par l'Établissement public d'aménagement (EPA) depuis le milieu des années 2000, a mis l'accent sur le fait que les cafés traditionnels ferment fréquemment à l'instar d'autres petits commerces ${ }^{13}$. Or le dynamisme commercial est désigné par les tenanciers de café comme primordial pour la santé de leur quartier d'implantation, en accord ici avec les aménageurs de la ville. Ils soulignent l'importance particulière des marchés comme en témoigne Morade : "Y'a très peu de cafés et ils ont tendance à disparaître. Sauf devant un marché. Il faut qu'il y ait un endroit où les gens puissent venir et le bistrot va profiter de cet afflux de personnes. »

Plus généralement, les cafetiers s'inquiètent des reconfigurations urbaines, non sans raison:

«Les commerces sont à la fois des marqueurs et des vecteurs du changement social dans les villes. Au sein des quartiers populaires centraux ou péricentraux, ils peuvent en particulier accompagner le processus de gentrification ou bien être menacés par lui. » (Berroir et al., 2015)

Ainsi, la Friterie se trouve aujourd'hui isolée dans le quartier ancien Saint-Jacques' situé au cœur de la ville, désormais embourgeoisé, mais continue à participer à l'animation du quartier en organisant des fêtes qui débordent sur la rue. Elle assure ainsi une certaine "continuité populaire», une "relative continuité du peuplement, des formes de sociabilité et des modes d'appropriation de l'espace local» (Giroud 2007, 2011). La "continuité populaire" désigne ici la manière dont les usagers issus des milieux populaires pratiquent les espaces après qu'ils aient été rénovés. Leur présence et leurs usages des lieux marquent l'espace. L'usage du bar s'affirme d'une certaine façon dans un rapport de force avec le voisinage. Formes, ambiances sonores, olfactives ou visuelles participent de ces promiscuités sensibles. Les vitrines tapageuses et enseignes acidulées des kebabs, côtoient difficilement la façade plus discrète des bars de quartier. Le stationnement ostentatoire et les conversations tapageuses sont perçus comme des incivilités par les voisins et les appropriations de l'espace public encouragent les crispations identitaires. Autant de problématiques récurrentes de la cohabitation entre des générations différentes d'habitants ou de clientèles, des usages populaires et ceux des couches supérieures, du partage d'un espace dit commun en négociation permanente. 
Illustration 18 : Anniversaire de Mohamed à la Friterie, janvier 2015

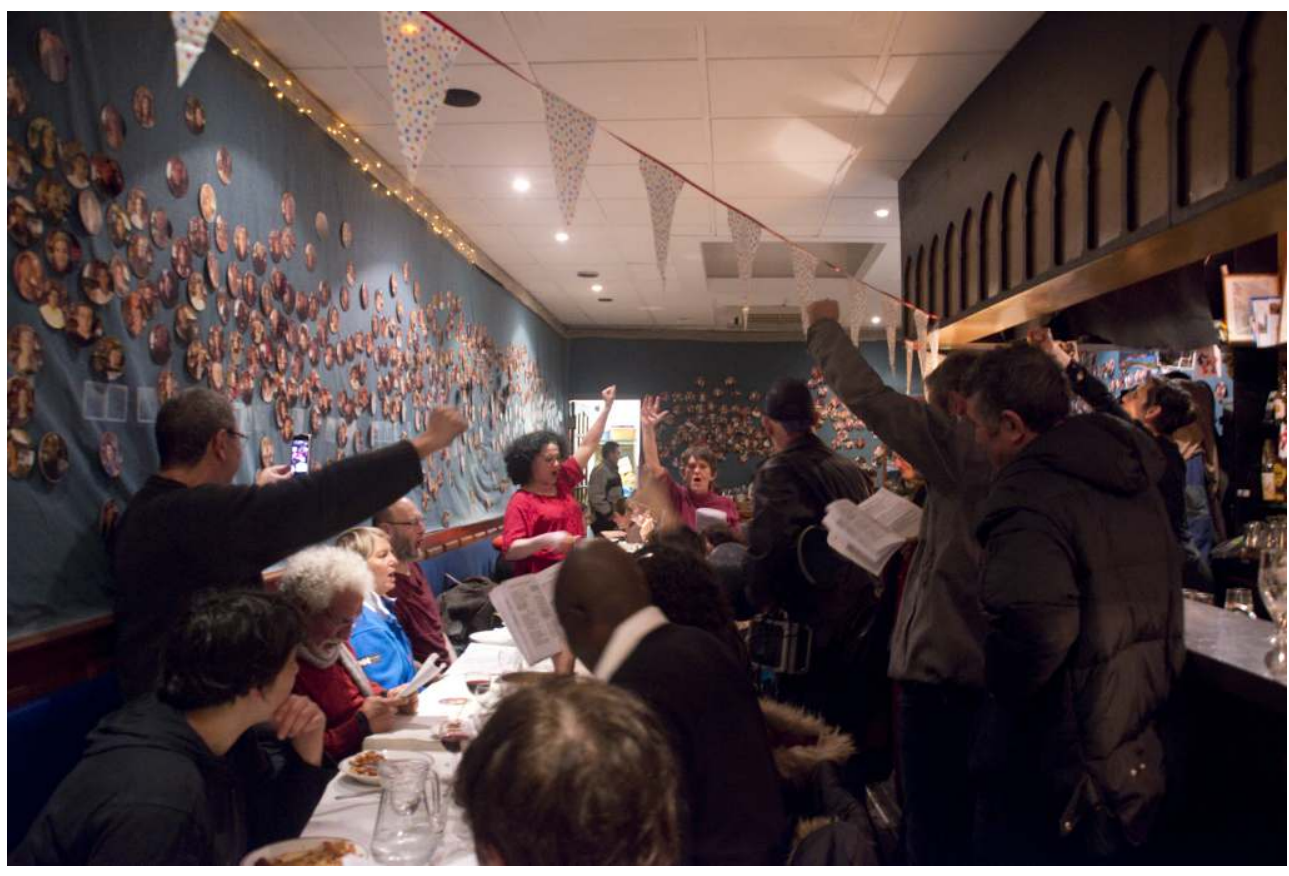

Source et copyright : ( ) Sandrine Binoux 2015.

Illustration 19 : Ambiance extérieure de la Friterie

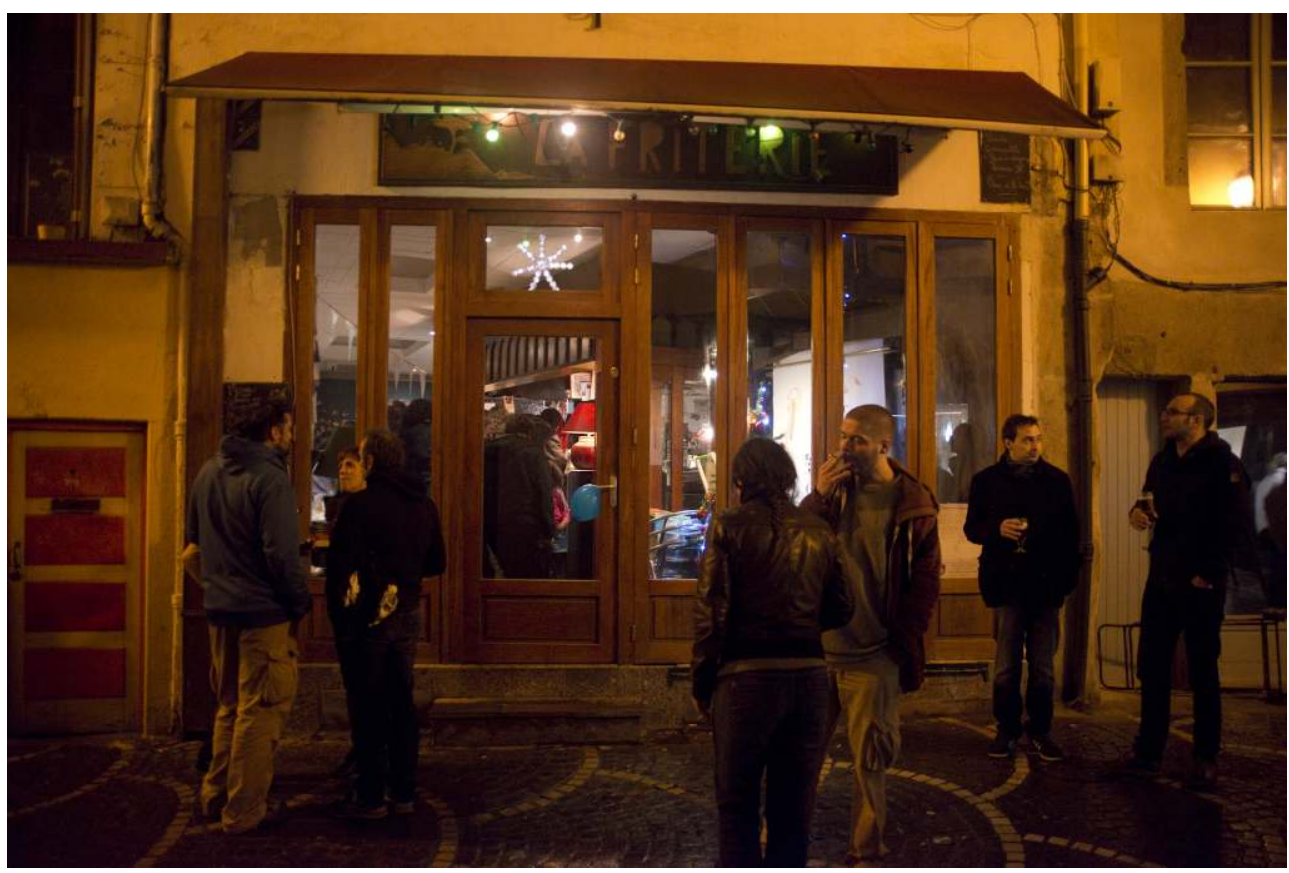

Source et copyright : ( ) Sandrine Binoux 2015.

Roland, comme Sandra, Marijo ou Mohamed et Louise, désirent nous dire au moment de l'enquête vouloir "passer la main", vendre leur établissement mais les acheteurs ne se bousculent pas. Le projet urbain en cours prévoit de son côté de nouvelles démolitions et réagencements urbains: ici le marché est déplacé, là d'importantes administrations ferment leur porte et des places de stationnement sont supprimées ; le théâtre Jean Dasté, 
emblématique du mariage heureux entre savant et populaire, déménage dans le quartier dit " créatif », à l'arrière de la Cité du design ; enfin les commerces voisins ferment les uns après les autres, comme dans bien d'autres villes (Oberti, 2016, p. 1 et p. 13). Ces craintes s'expriment plus facilement par les sentiments, les émotions liés à l'attachement à son établissement et sa survie que par une analyse de la politique urbaine conduite par la municipalité ou l'EPA.

« Finalement cette déco un peu kitch c'est... n'importe qui se sent à l'aise je trouve moi. Dans cette déco. Tu vois personne ne se la pète quoi. Et justement c'est dans des quartiers, c'est dans des endroits comme ça où tu te sens... c'est d'une simplicité extrême quoi. [Puis cela sonne comme un avertissement] Celui qui prendra après moi, s'il modifie le truc, s'il fait trop de travaux il perdra l'identité c'est clair mais il perdra beaucoup de clientèle !» (Sandra)

Illustration 20 : Intérieur du bar des Acacias

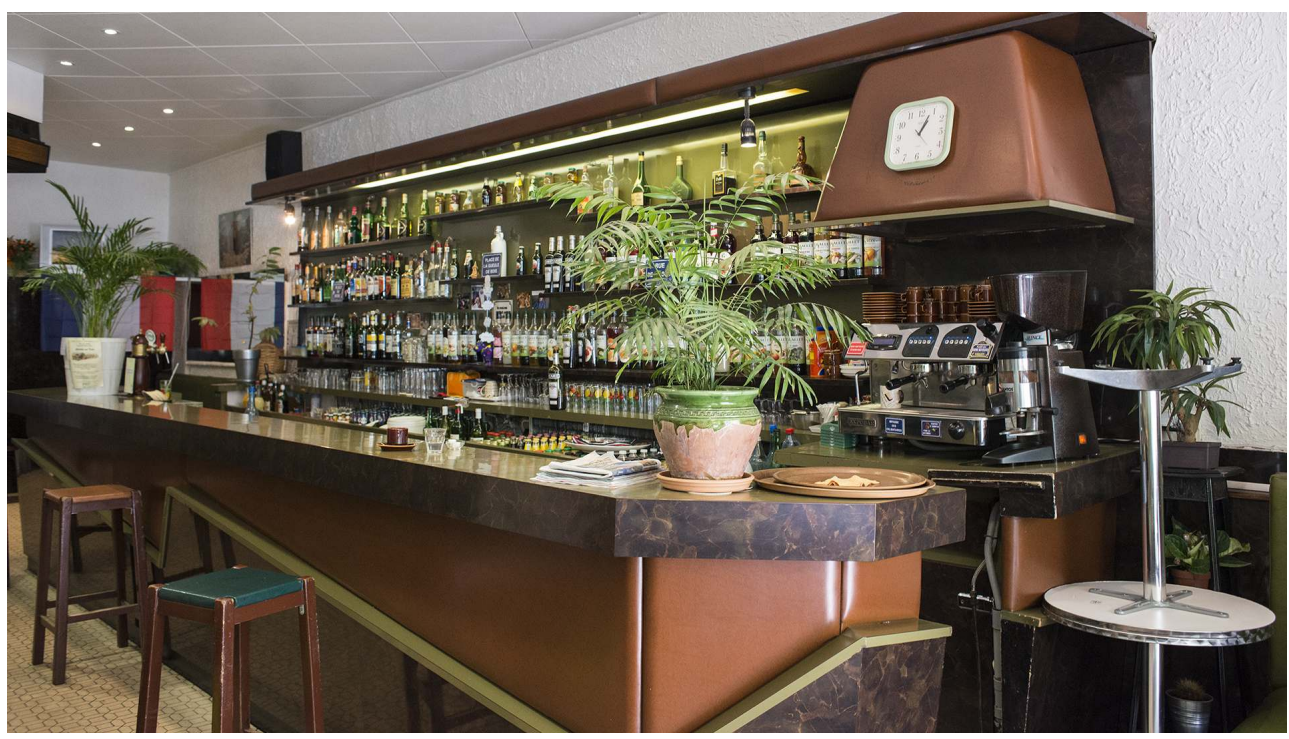

Source et copyright : (C) Sandrine Binoux 2015. 


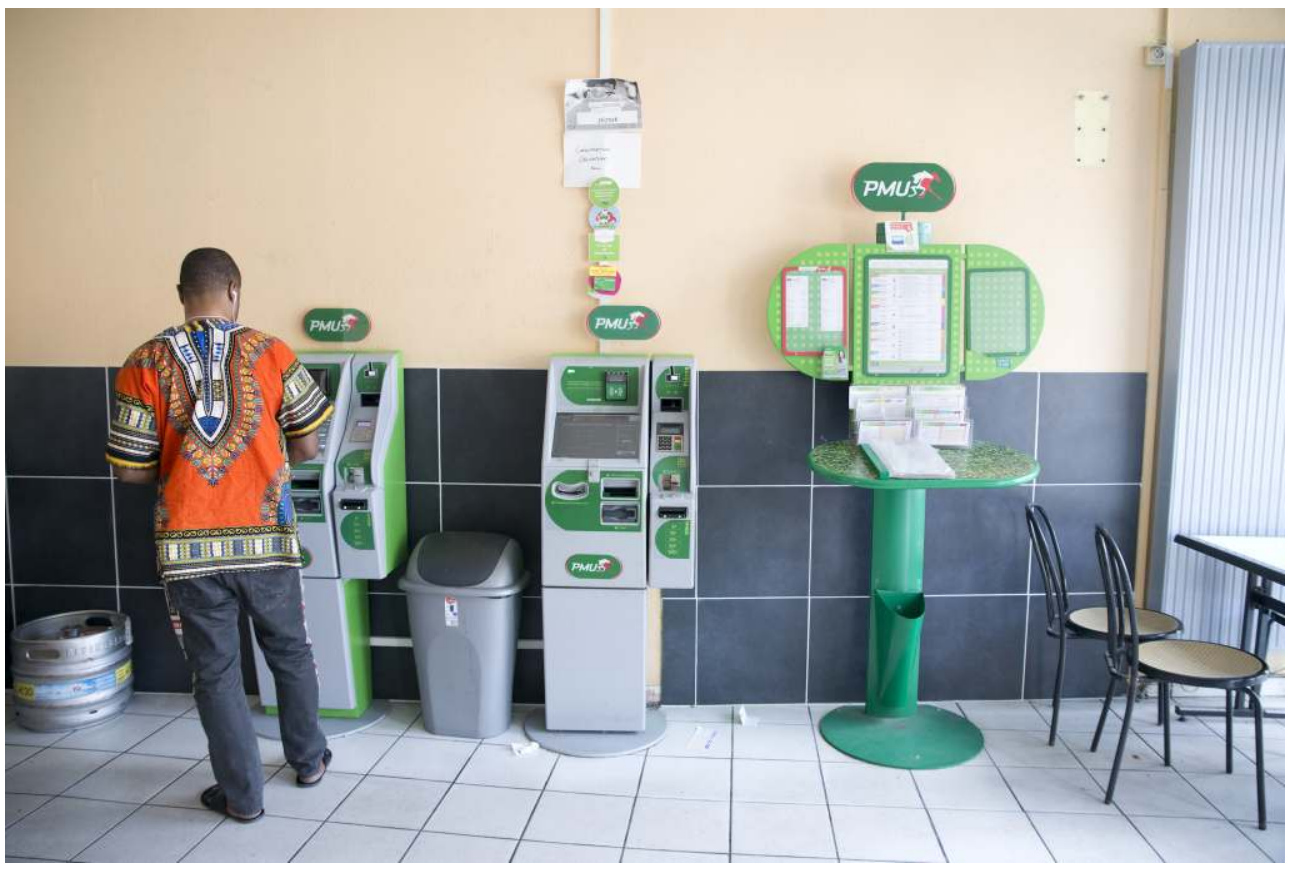

Source et copyright : ( ) Sandrine Binoux 2015.

Illustration 22 : Démolitions en cours du quartier Tarentaize

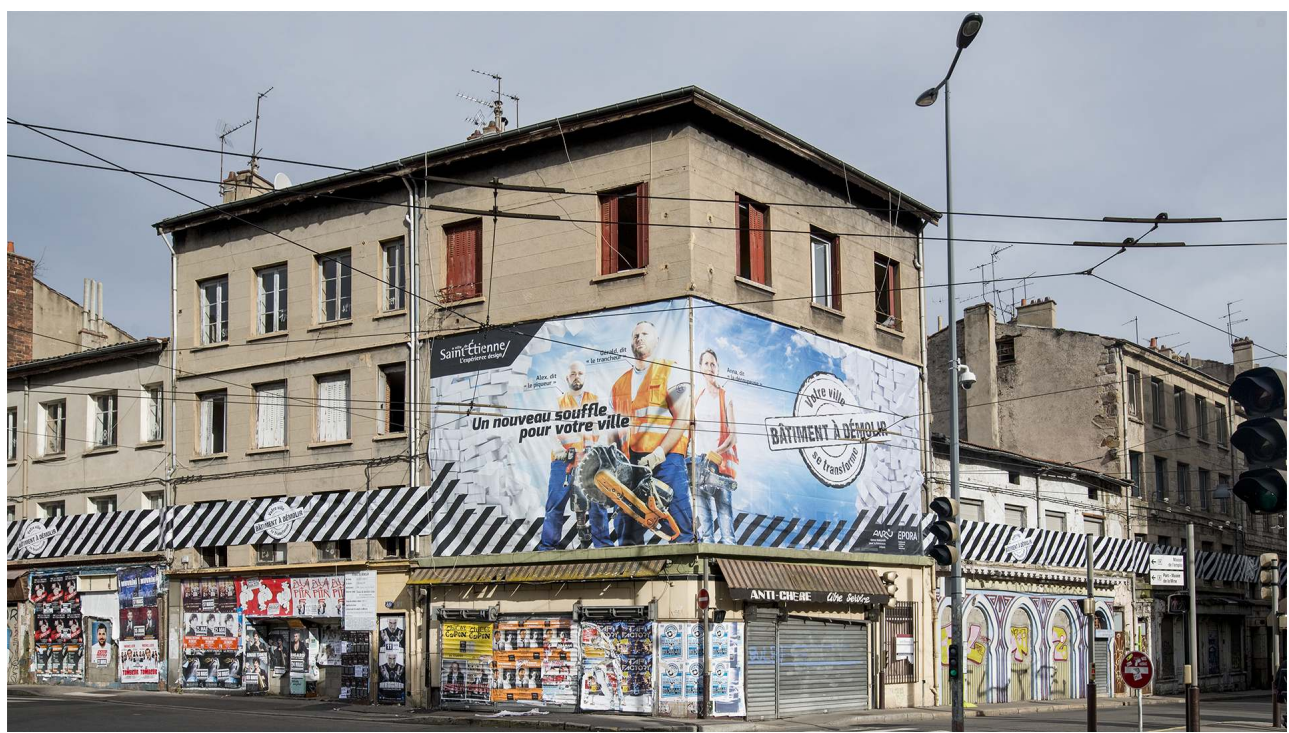

Source et copyright : ( ) Sandrine Binoux 2015.

Sortir le mobilier de la terrasse, ouvrir les portes, mettre de la musique, interpeller un passant, plaisanter, baisser le store, le remonter: les cafés populaires animent l'espace public des quartiers populaires par leur activité même. En se saisissant d'événements saisonniers ou ponctuels, ils participent à l'ambiance de la ville, à sa construction. Cependant, il est très rare qu'ils contribuent d'eux-mêmes aux grands événements officiels, telles que la Fête du Livre ou la Biennale internationale du Design. Ils tentent 
cependant, par des initiatives individuelles ou collectives, de ré-enchanter le commerce au niveau micro-local.

Les chercheurs peuvent d'ailleurs eux-mêmes participer de ces initiatives. Ainsi, la réalisation $\mathrm{du}$ film Sociologie de comptoirs a conduit les cafetiers à s'associer à une exposition aux archives municipales dans le cadre d'une valorisation de l'histoire du peuplement cosmopolite de la ville ${ }^{14}$. Corrélativement, le service Ville d'art et d'histoire et les archives ont décidé de mettre en place une saison culturelle pour valoriser ce type d'initiatives locales. Ainsi, durant neuf mois, des liens se sont tissés entre les différents protagonistes des projets. De leur participation, les patrons et patronnes de café renforcent leur détermination d'affirmation de l'identité sociologique des cafés: ouverture à l'étranger, rôle de renforcement des attachements entre différentes générations, rôle de rassemblement et de convivialité qu'il affirme comme relevant de la culture populaire. Ils y ont vu également le moyen de s'émanciper du discours dominant de la perte irréversible et d'adopter les registres de la culture et du patrimoine dans un geste politique de résistance ordinaire. Ceci leur donne le sentiment de pouvoir agir pour le maintien des quartiers populaires et d'en dessiner, pour une part, eux-mêmes les contours. Ils font ainsi la démonstration que les cafés sont des arènes ${ }^{15}$ et des acteurs potentiels de la fabrique de la ville d'aujourd'hui.

\section{Conclusion}

53 A l'heure où l'ambiance de l'espace public devient un enjeu fondamental pour l'aménagement urbain, il apparaît important d'expliciter la dimension qualitative et subjective que permettent ces cafés. Dans leurs murs et à leurs entours, ils peuvent affirmer leur fonction socio-spatiale singulière, en lien direct avec l'histoire des mémoires des cultures populaire, ouvrière et cosmopolite.

Ainsi les kébabs, bars, café-restaurants perdurent comme des lieux de sociabilité ordinaire et comme « ressource urbaine irremplaçable " (Berroir et al., 2015). A l'interface entre les cultures populaires d'ici et d'ailleurs et la modernité urbaine, ils assurent la survivance du droit à la ville de certaines catégories de population, moins jeunes, ou moins riches, moins cultivées que les populations visées par les investisseurs. Ceci en fait des lieux non de commémoration mais de production d'une mémoire parcellaire et bégayante, spontanée et contradictoire mais génératrice de liens sociaux, «milieux de mémoire" (Barrère \& Lévy-Vroelant, 2012), de reproductions et d'affirmations identitaires peu orthodoxes, par l'évocation d'une mémoire propre.

A l'issue de l'enquête et du travail filmique, le café est finalement devenu un outil d'analyse des effets du projet urbain ainsi qu'un terrain d'observation des résistances sociales, si difficiles à traquer dans leurs formes ordinaires. En devenant acteurs dynamiques de l'ambiance des espaces publics et la mémoire migratoire, patrons de café et clients cherchent à maîtriser la qualité des lieux, leur visibilité, tout en préservant leur avenir. Si les espaces intermédiaires de l'habitat peuvent être reconnus aujourd'hui comme les lieux de production du politique, les espaces intermédiaires de la ville et de la culture que sont les kébabs, restaurants et cafés des quartiers populaires, méritent également d'être appréhendés dans ce sens par les sciences humaines et sociales, les aménageurs, les architectes et les urbanistes. 


\section{BIBLIOGRAPHIE}

Arendt, Hannah. 1983 [1958]. Condition de l'homme moderne. Paris : Calmann-Lévy, coll. Pocket Agora.

Aslafy-Gauthier, Catherine. 2002. Mobilités marocaines via l'Espagne; de métissages ambulants en conquêtes clandestines. Thèse de doctorat. Toulouse : Université Toulouse le Mirail.

Augoyard, Jean-François. 2007. A comme Ambiance. Les cahiers de la recherche architecturale et urbaine. $\mathrm{n}^{\circ}$ 20-21, p. 33-37.

Bacqué, Marie-Hélène \& Levy, Jean-Pierre. 2007. Pour un droit à l'existence des quartiers populaires. Paris : La Découverte.

Balaÿ, Olivier. 2003. Trois utopies sonores pour la ville contemporaine. Espaces et sociétés. $\mathrm{n}^{\circ} 115$, p. 61-78.

Barrère, Céline \& Lévy-Vroelant, Claire. 2012. Hôtels meublés à Paris, enquête sur une mémoire de l'immigration. Paris : Créaphis, coll. Lieux habités.

Bazin, Hervé. 2009. Quels espaces populaires pour la culture ? Mouvement. $\mathrm{n}^{\circ} 57$. Paris : La Découverte. p. 57-66.

Berroir, Sandrine ; Clerval Anne ; Delage, Matthieu ; Fleury, Antoine ; Fol, Sylvie ; Giroud, Matthieu ; Raad, Lina \& Weber, Serge. 2015. Commerce de détail et changement social urbain : immigration, gentrification, déclin. EchoGéo. n 33 [En ligne]. Disponible sur : http:// echogeo.revues.org/14353 (consulté le 3 novembre 2016).

Charpy, Manuel. 2011. L'apprentissage du vide. Commerces populaires et espace public à Paris dans la première moitié du XIX ${ }^{\mathrm{e}}$ siècle. Espaces et sociétés. n 144-145, p.15-35.

Clavel, Maïté. 2002. Pour une recherche sur les pratiques des périurbains. Communications. $\mathrm{n}^{\circ} 73$, p. 203-216.

Clerval, Anne. 2011. L'occupation populaire de la rue : un frein à la gentrification? L'exemple de Paris intra-muros. Espaces et sociétés. $\mathrm{n}^{\circ}$ 144-145, p. 7-13.

Conord, Sylvaine (ed.). 2007. Arrêt sur images, Photographie et anthropologie. Ethnologie française. vol. XXXVII, $\mathrm{n}^{\circ} 1$, p. 11-21.

Curnier, Sonia. 2014. Programmer le jeu dans l'espace public ? Métropolitiques [En ligne]. Disponible sur : http://www.metropolitiques.eu/Programmer-le-jeu-dans-l-espace.html/ (consulté le 17 janvier 2016).

Dacheux, Eric. 2003. Un nouveau regard sur l'espace public et la crise démocratique. Hermès. $\mathrm{n}^{\circ}$ 36, p. 195-203.

Gauthier, Catherine (réal.). 2016. Sociologie de comptoirs, cafés cosmopolites [DVD]. Film photographique, photographies de Sandrine Binoux. Saint-Etienne : Centre Max Weber (CMW) / Association Migrations et Image Mémorielles (MIM). 35'.

Gauthier, Catherine. 2013. D'ici et de là-bas, St-Etienne / Timezrit, mémoires de mineurs. Saint-

Etienne : Puits Couriot, Parc musée de la mine, coll. Patrimoines du bassin de la Loire. vol. 3. 
Gauthier, Catherine. 2014. Les cafés de quartier comme lieux de mémoire. Écarts d'identité: Mémoire de l'accueil des étrangers. Lyon : La Fosse aux ours. p. 177-188.

Giroud, Matthieu. 2007. Résister en habitant ? Renouvellement urbain et continuités populaires en centre ancien (Berriat Saint-Bruno à Grenoble et Alcântara à Lisbonne). Thèse de doctorat, Poitiers :

Université de Poitiers [En ligne]. Disponible sur : http://tel.archives-ouvertes.fr/tel-00200103/fr/ (consulté le 4 mars 2016).

Giroud, Matthieu. 2011. Usages des espaces rénovés et continuités populaires en centre ancien. Espaces et sociétés. $\mathrm{n}^{\circ}$ 144-145, p. 37-54.

Grafmeyer, Yves. 2006. Sociologie urbaine. Paris : Armand Colin.

Grosjean, Michelle \& Thibaud, Jean-Paul (eds.). 2008. L'espace urbain en méthodes. Marseille : Parenthèses.

Hatzfeld, Hélène (ed.). 2015. Regards décalés sur les patrimoines silencieux. Pratiques interculturelles. Paris : Ateliers Henri Dougier.

Lemarchand, Nathalie. 2008. Géographie du commerce et de la consommation : les territoires $d u$ commerce distractif. Mémoire d'Habilitation à Diriger des Recherches, Paris : Université ParisSorbonne Paris IV.

Marcellini, Anne \& Miliani, Mahmoud. 1999. Lecture de Goffman. Corps et culture. $n^{\circ} 4$ [En ligne]. Disponible sur : http://corpsetculture.revues.org/641/ (consulté le 3 octobre 2017).

Oberti, Marco. 2016. Faire émerger la mixité. In Maurin, Louis \& Schmidt, Nina. Que faire contre les inégalités ? 30 experts s'engagent. Rapport de recherche. Paris : Observatoire des inégalités.

Pétonnet, Colette. 1987. L'anonymat ou la pellicule protectrice. Le temps de la réflexion. vol. VIII (La ville inquiète), p. 247-261.

Pichon, Pascale ; Herbert, Fanny \& Perdrix, Alisonne. 2015. Atlas des espaces publics, Saint-Étienne une ville laboratoire. Saint-Etienne : PUSE.

Raulin, Anne. 1986. Mises en scène des commerces maghrébins parisiens. Terrain. nº 7, p. 24-33.

Raulin, Anne. 2001. Anthropologie urbaine. Paris : Armand Colin.

Rétière, Jean-Noël. 2003. Autour de l'autochtonie. Réflexions sur la notion de capital social populaire. Politix. $\mathrm{n}^{\circ}$ 63, p.121-143.

Sauvadet, Thomas \& Bacqué, Marie-Hélène. 2011. Éditorial. Les pratiques populaires de l'espace. Espaces et sociétés. $\mathrm{n}^{\circ}$ 144-145, p. 7-13.

Siblot, Yasmine ; Cartier, Marie ; Coutant, Isabelle ; Masclet, Olivier \& Renahy, Nicolas. 2015. Sociologie des classes populaires contemporaines. Paris : Armand Colin.

Steiner, Anne \& Conord, Sylvaine. 2012. Portrait d'un bistrot des faubourgs : Le Mistral. Ethnologie française. vol. 42, $\mathrm{n}^{\circ}$ 3, p. 475-476.

Suzanne, Gilles. 2009. Musiques d'Algérie, mondes de l'art et cosmopolitisme. Revue européenne des migrations internationales. vol. 25, $\mathrm{n}^{\circ} 2, \mathrm{p} .13-32$.

Tapie, Guy. 2014. Sociologie de l'habitat contemporain, vivre l'architecture. Marseille : Parenthèses.

Tarrius, Alain. 1997. Fin de siècle incertaine à Perpignan. Perpignan : éditions Trabucaire.

Tarrius, Alain \& Missaoui, Lamia. 2001. Les nouveaux cosmopolitismes. Mobilités, identités et territoires. La Tour d'Aigues : l'Aube. 
Tebbakh, Sonia. 2007. Une transmission discrète et fragmentaire. De l'histoire migratoire dans les familles maghrébines. Temporalités [En ligne]. Disponible sur : http:// temporalites.revues.org/200/ (consulté le 5 mai 2017).

Thibaud, Jean-Paul. 2015. L'ordinaire des ambiances urbaines. In : Pichon, Pascale ; Herbert, Fanny \& Perdrix, Alissone. Atlas des espaces publics, Saint-Étienne une ville laboratoire. Saint-Étienne : PUSE, p. 24-29.

Toubon, Jean-Claude \& Messamah, Khélifa. 1991. Centralité immigrée, le quartier de la Goutte d'Or. 2 vol. Paris : L'Harmattan-Ciemi.

Verret, Michel. 1995. L'espace ouvrier. Paris : L'Harmattan.

\section{NOTES}

1. Cet article s'appuie en partie sur deux communications : Gauthier, Catherine. 2016. Les cafés de quartiers et l'immigration, recherche pour un film documentaire de photos. CR 02: Sociologie urbaine: villes, sociétés et action publique. $\mathrm{XX}^{\mathrm{e}}$ congrès de l'AISLF, 4-8 juillet 2016. Montréal ; Gauthier, Catherine. 2017. Images et sons de la mémoire des lieux. Colloque International Les formes de l'enquête. CIEREC, Université Jean Monnet. 6-8 avril 2017. Saint-Etienne.

2. Recherche financée par l'appel à projet de la DRAC et la Région Rhône-Alpes « Mémoires du XX e siècle » inscrite dans l'exposition municipale «Saint-Étienne cosmopolitaine, des migrations dans la ville », présentée en 2015-2016.

3. Cette démarche avoisine d'autres travaux portant sur des objets similaires, où le dispositif d'enquête a aussi associé sociologue et photographe (Steiner \& Conord, 2012 ; Conord, 2007 ; Barrère \& Lévy-Vroelant, 2012).

4. Termes à la mode dans le design et marketing à l'œuvre dans le projet urbain signé par BIG, Superflex et Topotek 1 pour la ville de Copenhague, l'un des dix-neuf finalistes du prix Aga Khan Award for Architecture. Disponible sur: http://www.floornature.eu/superkilen-copenhagueprojet-urbain-big-superflex-topotek-1-11892/(consulté le 6 mai 2017).

5. Cette démarche s'inscrit dans la tradition de l'école de Chicago et la pluridisciplinarité des approches actuelles de l'espace public urbain.

6. Selon la méthode ethnographique, l'échantillonnage est constitué à partir de critères de diversification, en fonction des hypothèses, pour obtenir des exemples d'une grande diversité à l'égard du thème de l'étude (sexe, âge, origine géographique de l'immigration du patron et/ou de ses parents, origine géographique de la clientèle, type de services annoncés par le bar comme par exemple PMU ou petite restauration...).

7. Mémoire de l'immigration et mémoire migratoire sont à distinguer. La première désigne la mémoire portée ou non par une société de l'histoire de l'immigration. La seconde désigne les processus de transmission d'une mémoire, de parcours et d'expériences migratoires au sein d'un groupe donné (Tebbakh, 2007).

8. Les noms indiqués pour les personnes et les lieux sont réels, tout comme dans le film. Le montage de ce dernier cependant brouille un peu les pistes en croisant image et son de façon non littérale.

9. Le commerce ethnique et son quartier d'implantation jouent ce rôle de connexion entre territoire circulatoire et société d'accueil (Aslafy-Gauthier, 2002 ; Gauthier, 2013).

10. Voir la carte des quartiers étudiés.

11. Notre usage ici des notions de "vie quotidienne » et de "culture populaire » est étroitement associé à la « vie de café » comme M. de Certeau en montre l'association dans 
la « vie de quartier ", entre bricolage, tactique et construction de la ville à l'échelle de la rue et au rythme de la marche.

12. Sur la qualité acoustique des lieux et la conscience de leur importance dans les sociabilités, voir (Augoyard \& Balä̈, 2003).

13. Sur ce sujet, voir le travail effectué par les associations Rue du Développement Durable et Carton Plein à Saint-Étienne dans les quartiers du Crêt-de-Roch et de Jacquard, en partie retracés dans l' Atlas des Espaces Publics. Saint-Etienne une ville laboratoire (Pichon et al., 2015).

14. «Saint-Étienne cosmopolitaine, des migrations dans la ville », présentée en 2015-2016 par les Archives municipales de Saint-Étienne et le service Ville d'Art et d'Histoire de la ville de SaintÉtienne. Le livret «Laissez-vous conter Saint-Étienne cosmopolitaine », édité en 2016, retrace le contenu de l'exposition et les projets en cours.

15. Dans ce contexte local, les remarques d'Éric Dacheux sur la dimension politique des espaces frontières entre l'espace public et la sphère privée, inspirant l'économie sociale et solidaire et les démarches participatives sont tout à fait appropriées (Dacheux, 2003, p. 201-202).

\section{RÉSUMÉS}

Étudiant la mémoire de l'immigration dans les quartiers populaires des centres anciens de Saint Etienne, Catherine Gauthier, accompagnée de la photographe Sandrine Binoux, saisit ambiances et portraits dans les cafés cosmopolites de cinq quartiers. Il en résulte un film photographique de 35 minutes, retraçant souvenirs et ambiances actuelles de ces établissements. L'article montre comment la rue, l'usine, la vie migratoire les ont façonnés, puis comment leur activité déborde à son tour sur l'espace public par une animation très spontanée. D'abord ressource urbaine pour les primo-arrivants, les cafés peuvent devenir aujourd'hui des emblèmes de la mémoire locale. Enquête et film permettent de visiter l'histoire des centralités commerciales en déclin et de rendre compte des traces encore vivantes d'une culture vernaculaire de la mixité socioculturelle.

Studying immigrants' memories in the popular districts of downtown old Saint-Etienne, Catherine Gauthier, sociologist, and Sandrine Binoux, photographer, capture atmospheres and portraits in the cosmopolitan cafés of five districts. This has resulted in a 35-minute film, retracing memories and present atmospheres of these establishments. The article shows how the street, the factory, and migratory life have shaped them, and then how their activity flows over into the public space very spontaneously. Initially an urban resource for newcomers, today the cafés can become an emblem of local memory. The research and the film allow us to visit the history of the declining commercial central locations, and to chart the traces of a vernacular culture of socio-cultural diversity.

\section{INDEX}

Keywords : popular districts, cosmopolitan cafés, migratory memory, ambiances, public space Mots-clés : quartiers populaires, cafés cosmopolites, mémoire migratoire, ambiances, espace public 


\section{AUTEUR}

\section{CATHERINE GAUTHIER}

Catherine Gauthier est socio-anthropologue, maître assistante associée en sciences humaines et sociales à l'ENSA Saint-Etienne et à l'ENSA Clermont-Ferrand, et chercheure associée au Centre Max Weber, UMR CNRS 5283. Ses recherches portent plus spécifiquement sur les mémoires migratoires et urbaines en tant que manifestations d'une culture commune. Elles s'appuient sur des méthodes audio-visuelles tout en développant une démarche immersive et sensible catherinegauthier42@yahoo.fr 